\title{
DONNÉES BIBLIOGRAPHIQUES SUR L'ÉLEVAGE DES JEUNES STADES ET LA BIOLOGIE DES CORÉGONES
}

\author{
A. MACK *, R. BILLARD * *
}

* Pisciculture de Villerin, 21530 LA ROCHE-EN-BRENIL

* *.N.R.A., Département d’Hydrobiologie - 78850 JOUY-EN-JOSAS.

\begin{abstract}
RESUMÉ
Une revue sommaire de la bibliographie portant sur quelques aspects de la biologie et de l'élevage du corégone fait l'objet de cet article. Les problèmes de systématique sont brièvement exposés. La présentation de la biologie de l'espèce (gamétogenèse. fécondité, embryogenèse, croissance, exigences de l'environnement, etc.) est développée en relation avec les problèmes d'élevage posés par ces espèces: stockage des géniteurs, reproduction, insémination artificielle, incubation, élevage larvaire dans diverses conditions, élevage des estivaux, etc. Les possibilités d'utiliser ces espèces pour l'élevage en étang ou pour le pacage lacustre sont examinées.
\end{abstract}

\section{SUMMARY}

Bibliographic data on the rearing of coregonid larvae and alevins, and coregonid biology.

This article gives a brief review of the bibliography on some aspects of the biology and culture of coregonids. Systematic problems have been concisely presented. The biology of coregonids (gametogenesis, fecundity, embryology, growth, environmental requirements, etc) is developed in relation with the problems of rearing the species : stocking of brood fish, reproduction, artificial insemination, incubation, alevin raising in various conditions, raising of yearlings. The possibilities of using these species for pond culture or lake ranching are discussed.

\section{INTRODUCTION}

Dans de nombreux lacs, plusieurs espèces de corégones font l'objet d'une pêche commerciale importante qui est souvent excessive, entraînant une diminution des stocks. On essaie, alors, de pratiquer des repeuplements et on procède traditionnellement au déversement d'ceufs embryonnés ou de larves vésiculées le plus souvent originaires de parents prélevés dans le même lac, à moins que la reproduction naturelle ne puisse plus avoir lieu. Les avantages de l'opération ne sont pas évidents car les chances de survie des larves déversées ne sont pas meilleures que celles issues de reproduction naturelle (le taux de survie étant dans ce cas de l'ordre de 1 a $2 \%$, EINSELE 1965, BENAROUS 1981).

D'autre part, l'efficacité de ces repeuplements n'a jamais été bien démontrée et CHRISTIE (1963) n'a pas observé d'amélioration notable du recrutement après déversement de juveniles.

Une autre approche consiste à ne lâcher dans les lacs que des sujets prégrossis qui résistent mieux à la prédation et dont la taille lors du lâcher est de $5-7 \mathrm{~cm}$ et $2 \mathrm{~g}$. Ce prégrossissement se pratique pendant plusieurs semaines, soit en bassins ou étangs d'alevinage, soit en cages immergées dans le lac même où les juvéniles seront libérés. Cette approche est maintenant pratiquée dans divers pays comme la Pologne. l'Allemagne, la Suisse et est en projet en France pour le Lac Léman. Elle peut prendre des formes plus ou moins élaborées; dans le cas le plus simple, les géniteurs sont prélevés dans le lac; dans un cas plus complexe, on se dote d'un stock de géniteurs élevés dans divers plans d'eau facilement pêchables, ce qui revient à pratiquer une véritable pisciculture

Dans le présent document, nous ferons le point bibliographique (non exhaustif, car la littérature est très vaste) sur l'élevage des jeunes stades et sur quelques éléments de biologie générale, en rapport avec la pisciculture (gamétogenèse, fécondité, insémination artificielle, etc.). En outre, le problème complexe de la systématique sera sommai- 
rement abordé. Parmi les bibliographies importantes sur les corégones, nous citerons MARSHALL et WOODS (1971), SWANN et DONALDSON (1980).

\section{A - systematique du corégone}

Les données que nous rapportons dans ce paragraphe se réfèrent surtout à l'article de BEHNKE (1970). La famille des Salmonidés se divise en trois sous-familles : les Salmonidés (truites, saumons), les Corégonidés et les Thymallinés (ombre). La sousfamille des Corégonidés se divise en 3 genres : Prosopium. Stenodus et Coregonus.

La figure 1 présente un essai de classification des différentes espèces du genre Coregonus. Mais il faut bien dire qu'il règne la plus grande confusion dans la détermination et le classement de ces espèces. A cela, BEHNKE (1970) donne deux raisons :
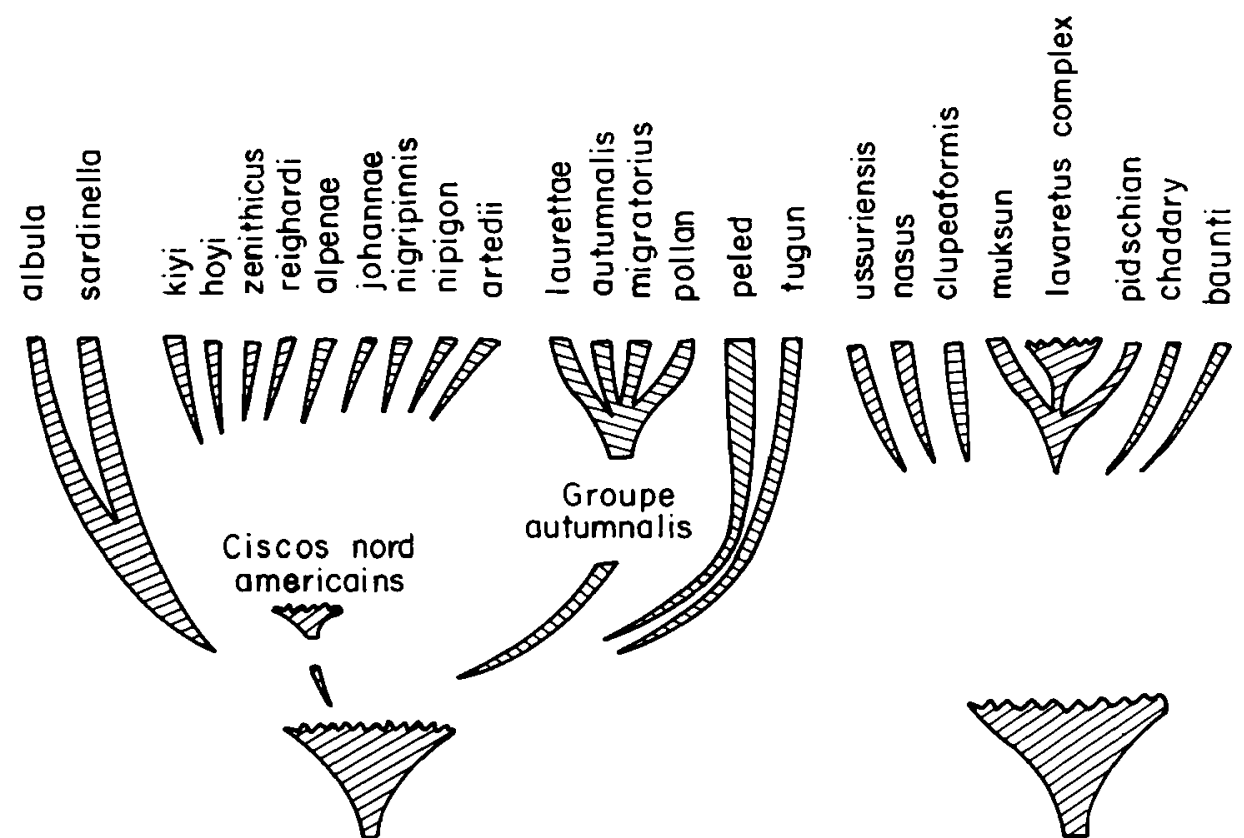

Les ciscos

sous genre leucichthys
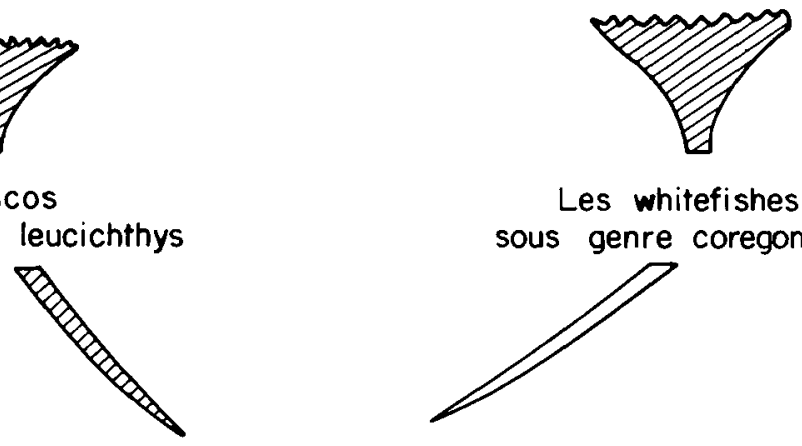

Les whitefishes sous genre coregonus

Figure 1 : Relations supposées entre les différentes especes du genre Coregonus (d'après BEHNKE, 1970). Il faut préciser qu'il existe une forte variabilité des caractères entre espèces et même à l'int ́́rieur d'une même espèce. Des clés systématiques sont aussi donnees par SCOTT et CROSSMAN (1973) pour les corégonidés du Canada.

- la plasticité du phénotype (en effet, pour un même génotype, la mor:;hoijgia du poisson peut être complètement différente suivant les conditions ou milieu ou d'élevage).

- un comportement sur les lieux de reproduction admettant diverses populations de cette espece.

TODD et SMITH (1980) ont eux aussi noté des difficultés dans la classification des Ciscos des grands lacs américains et prenant le cas de $C$. zenithicus, qui présente une forte variabilité morphologique, constatent que saison de ponte et isolation géographi- 
que agissent de façon similaire dans la différenciation des populations de corégonidés et que les populations frayant à différentes saisons ne constituent pas nécessairement des espèces différentes.

D'après BEHNKE (1970), la Fera du lac Léman se situe dans l'espèce Lavaretus complex. SVARDSON (1949-1957) propose une superespèce, Lavaretus, contenant 5 espèces. Mais la distinction n'est fondée que sur une différence du nombre de branchiospines, ce qui est contesté par BEHNKE (1970) qui propose, lui, une seule espèce, le "Coregonus lavaretus complex".

\section{B - INCUBATION DES CUFS}

\section{1) Insémination artificielle}

Les techniques actuellement pratiquées ne sont pas très performantes. STEFFENS (1978) préconise la technique de la fécondation semi-humide, c'est-à-dire que les œufs sont recueillis dans un récipient avec le liquide ovarien, et le sperme est rajouté ensuite, puis mélangé. On ajoute l'eau et on brasse le tout avec précaution. Il faut laisser reposer pendant 10 a 15 minutes avant de rincer plusieurs fois avec de l'eau fraîche avant le passage en incubateur (bouteille de Zug ici, fig. 2), afin d'éviter que les œufs se collent entre eux et se trouvent en anoxie.

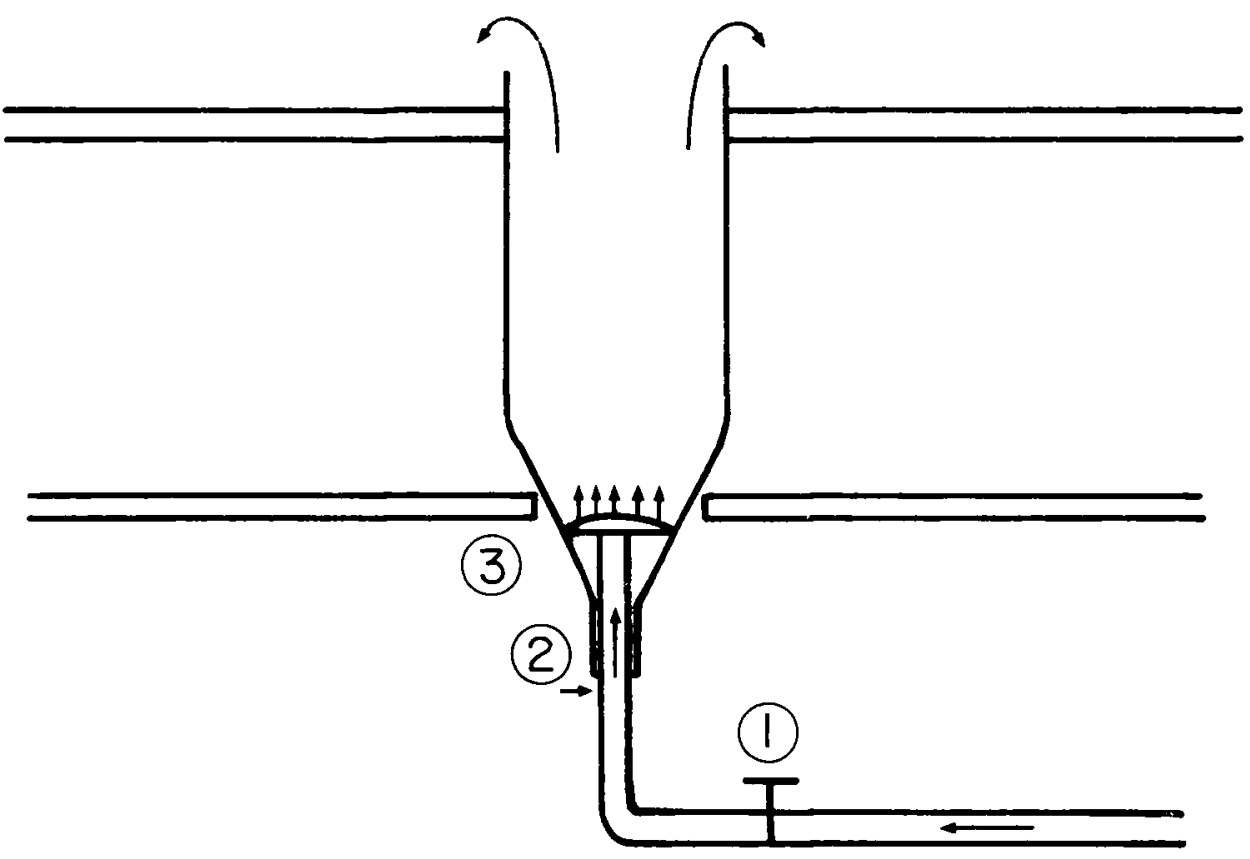

Figure 2: Schéma d'une bouteille de Zug. L'eau circule de bas en haut provoquant ainsi le mouvement continu des ceufs.

1 : robinet

2 : manchon démontable permettant de retirer la bouteille avec son contenu.

3 : admission par une pomme de douche permettant une meilleure répartition de l'eau.

Par température très froide (gel), on peut réaliser la fécondation par méthode humide. Les œufs sont recueillis dans un bol avec de l'eau, et la laitance est aussitôt ajoutée, puis brassée sans interruption. On évite ainsi les dommages dus au froid sur les œufs. 


\section{2) Transport}

Dans certains cas, l'insémination peut se pratiquer loin du site d'incubation et ilya lieu alors de transporter les œufs fraîchement fécondés vers l'écloserie. Cela est possible dans un sac plastique rempli d'eau ou dans des pots au-dela de plusieurs heures. La quantité d'œu's ne doit pas dépasser plus de $30 \%$ de la quantité d'eau. Le transport des œufs non fécondés est à éviter (STEFFENS, 1978).

\section{3) Température}

Le nombre de degrés-jour entre insémination et éclosion varie suivant la tempéra ture d'incubation, le maximum se situant vers 5,6 à $6^{\circ} \mathrm{C}$ (500 à 520 degrés-jour) (Tabl. 1).

(A)

\begin{tabular}{|l|c|c|c|c|c|}
\hline Température & $2^{\circ} \mathrm{C}$ & $4^{\circ} \mathrm{C}$ & $5.9^{\circ} \mathrm{C}$ & $7.8^{\circ} \mathrm{C}$ & $10^{\circ} \mathrm{C}$ \\
\hline Coregonus clupeaformis & 305 & 446 & 500 & 460 & 417 \\
\hline
\end{tabular}

(B)

\begin{tabular}{|l|c|c|c|c|c|c|c|}
\hline Température & $0.5^{\circ} \mathrm{C}$ & $1,7^{\circ} \mathrm{C}$ & $3,4^{\circ} \mathrm{C}$ & $5,6^{\circ} \mathrm{C}$ & $7.7^{\circ} \mathrm{C}$ & $8,9^{\circ} \mathrm{C}$ & $10^{\circ} \mathrm{C}$ \\
\hline Coregonus artedii & 118 & 319 & 500 & 521 & 460 & 418 & 382 \\
\hline
\end{tabular}

Tableau 1 : Temps d'incubation en degrés-jour du Coregonus clupeaformis (A) et du Coregonus artedii (B) en fonction de la température d'incubation. (COLBY ot BROOKE, 1973 ; BROOKE, 1975).

En ce qui concerne le corégone du lac Léman que nous étudions, $50 \%$ des œufs éciosent après 345 degrés-jours pour une température d'incubation de $4,9^{\circ} \mathrm{C}$. Cette espèce semble donc demander une période d'incubation plus courte que Coregonus clupeaformis ou Coregonus artedii.

En outre, la température d'incubation ne doit pas être trop élevée. Ainsi, chez Coregonus clupeaformis, si l'on obtient une éclosion en 182 jours à $0,5^{\circ} \mathrm{C}$ et en 41 jours à $10^{\circ} \mathrm{C}$, la meilleure température d'incubation semble être $4^{\circ} \mathrm{C}$ (111 jours) où l'on arrive à des chiffres de $73 \%$ de survie, $(2,8 \%$ d'alevins anormaux) et $13,5 \mathrm{~mm}$ à l'éclosion. En effet, pour une température d'incubation inférieure ou supérieure à $4^{\circ} \mathrm{C}$, ces trois paramètres varient défavorablement (BROOKE, 1975) (tableau 2). La température

\begin{tabular}{|c|c|c|}
\hline \multirow[t]{2}{*}{ ESPECES } & \multicolumn{2}{|c|}{ TEMPERATURES D'INCUBATION $\left({ }^{\circ} \mathrm{C}\right)$} \\
\hline & LIMITES & OPTIMUM \\
\hline $\begin{array}{l}\text { Cisco } \\
\text { C. ripus } \\
\text { C. peled } \\
\text { C. lavaretus ludoga } \\
\text { C. lavaretus sevan } \\
\text { C. lavaretus beari } \\
\text { C. lavaretus marenoides } \\
\text { Stenodus leucichthys leucichthys } \\
\text { Stenodus leucichthys nelma } \\
\text { C. autumnalis } \\
\text { C. nasus } \\
\text { C. muksun }\end{array}$ & $\begin{array}{l}1,5-7 \\
1,2-6 \\
0.1-5 \\
0,1-8 \\
0,1-8 \\
0,1-6 \\
0.1-6 \\
0.1-6 \\
0,1-8 \\
0,1-5 \\
0.5-1,7 \\
0.5-2\end{array}$ & $\begin{array}{l}2,5 \cdot 5 \\
1,5-4 \\
1,5 \cdot 4,3 \\
1,5-3 \\
1,2-4 \\
1,5-5 \\
0,5 \cdot 1,5\end{array}$ \\
\hline
\end{tabular}

Tableau 2 : Températures d'incubation (limites extrêmes et optimum) pour quelques espèces de coregonidae (d'apres CHERNYAEV, 1982). 
affecte aussi la morphogenèse (tableau 3). On n'obtient d'ailleurs pas d'éclosion lorsque la température d'incubation est de $0^{\circ} \mathrm{C}$ ou de $12^{\circ} \mathrm{C}$. Suivant les 20 premières divisions du développement embryonnaire énoncées par COLBY et BROOKE (1973) (tableau 4), l'œuf atteint le stade 3 a $0^{\circ} \mathrm{C}$ avant de mourir, et à $12^{\circ} \mathrm{C}$, ils sont très déformés au stade 12 et meurent avant ou pendant l'éclosion (fig. 3). On note également que l'intervalle entre le premier éclos et le dernier est plus court à $4^{\circ} \mathrm{C}$ qu'à $2^{\circ} \mathrm{C}$ (BIDGOOD, 1974).

\begin{tabular}{|c|c|c|c|}
\hline $\begin{array}{c}\text { Température } \\
\text { d'incubation }\left(^{\circ} \mathrm{C}\right)\end{array}$ & $\begin{array}{c}\text { Nombre de } \\
\text { myotomes }\end{array}$ & $\begin{array}{c}\text { Longueur totale } \\
(\mathrm{mm})\end{array}$ & $\begin{array}{c}\text { Poids } \\
\text { (mg) }\end{array}$ \\
\hline $1.8(0-7,2)$ & $58,2(56-63)$ & $13.2(11,3-14,1)$ & 8.8 \\
$5,1(3,6-7,9)$ & $55,1(50-62)$ & $12,7(11,2-13,1)$ & 7,6 \\
$8,4(7,5-9,4)$ & $54,9(50-60)$ & $11,1(9,5-12,5)$ & 6,5 \\
\hline
\end{tabular}

Tableau 3 : Influence de la température d'incubation sur la morphogenèse de l'embryon de C. autumnalis (d'après CHERNYAEV, 1982) () extrêmes.

\begin{tabular}{|c|c|c|}
\hline Stades & Description & $\begin{array}{c}\text { Age en jours } \\
\left(5,6^{\circ} \mathrm{C}\right)\end{array}$ \\
\hline $\begin{array}{c}1 \\
2 \\
3 \\
4 \\
5 \\
6 \\
\\
7 \\
8 \\
9 \\
10 \\
11 \\
12 \\
13 \\
14 \\
15 \\
16 \\
\\
17 \\
18 \\
19 \\
20\end{array}$ & $\begin{array}{l}\text { Blastoderme enveloppant } 1 / 3 \text { du vitellus. } \\
\text { Blastoderme enveloppant la moitié du vitellus. } \\
\text { Blastoderme enveloppant les } 2 / 3 \text { du vitellus. } \\
\text { Formation de la raie embryonnaire. } \\
\text { Présence des optiques primordiales. } \\
\text { L'embryon s'étend autour du vitellus (occupe la moitié de la } \\
\text { courbure du vitellus). } \\
\text { Deux globules optiques se forment avec les lentilles primordiales. } \\
\text { La queue commence à être marquée par un repli. } \\
\text { L'embryon occupe les } 2 / 3 \text { de la courbure du vitellus. } \\
\text { Queue repliée, mouvement du corps visible. } \\
\text { Premiers pigments des yeux visibles. } \\
\text { Pigments des yeux réellement visibles sans grossissement. } \\
\text { Ebauche des nageoires pectorales. } \\
\text { Nageoires pectorales formees. } \\
\text { Circulation du sang visible dans les branches des veines latérales } \\
\text { vitellines du cœeur ou du vitellus } \\
\text { Formation de la nageoire caudale. Vitellus avec un ou deux gros } \\
\text { globules gras. } \\
\text { Chromatophores étoilés sur une double rangée le long de tout le } \\
\text { corps, sauf la tête. } \\
\text { La nageoire pectorale forme une lance. Mouvement de la nageoire } \\
\text { visible. } \\
\text { Chromatophores étoilés sur la tête. } \\
\text { Agitation constante de la nageoire pectorale. } \\
\text { Mouvements des yeux } \\
\text { Eclosion. }\end{array}$ & $\begin{array}{l}8 \\
8,5 \\
9 \\
10,5 \\
12 \\
13,5 \\
16 \\
18 \\
18,5 \\
20,5 \\
24 \\
27 \\
29,5 \\
\\
31 \\
\\
33 \\
40 \\
\\
48,5 \\
51 \\
58 \\
65 \\
93,2\end{array}$ \\
\hline
\end{tabular}

Tableau 4 : Stades de développement embryonnaire de C. artedii selon COLBY et BROOKE (1975).

\section{4) Demande en oxygène}

L'œuf en lui-même a besoin de peu d'oxygène. Mille œufs de Coregonus autumnalis migratorius consomment au stade de la segmentation ou de la gastrulation $0,1 \mathrm{mg}$ d'oxygène en une heure, au stade de la pigmentation des yeux $0,17 \mathrm{mg}$ et avant éclosion $0,29 \mathrm{mg}$ (CHERNYAEV, 1977-1982) (tableau 5, fig. 4). Mais l'eau doit être à saturation car la configuration de l'œuf est défavorable à l'entrée d'oxygène. Il faut au moins $8 \mathrm{mg} / \mathrm{I}$ au stade ceillé ( $5^{\circ} \mathrm{C}-65 \%$ de la saturation) et pendant tout le reste du développement (FLUCHTER, 1980). 
Figure 3 :

Vitesse de segmentation du blastodisque d'coufs de C. autumnalis incubés à deux températures constantes 3 et $0,5^{\circ} \mathrm{C}$ (d'après CHERNYAEV,1982).

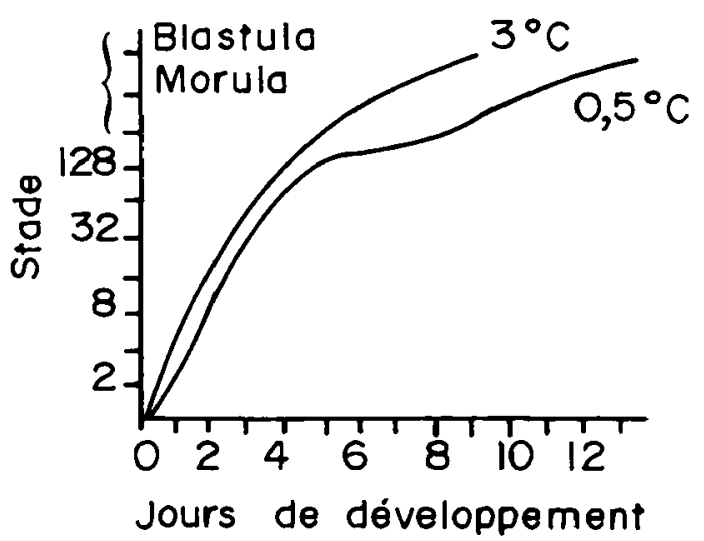

\begin{tabular}{|c|c|c|c|}
\hline $\begin{array}{c}\text { Jours de } \\
\text { développement }\end{array}$ & $\begin{array}{c}\text { Stade de } \\
\text { l'embryogenèse }\end{array}$ & $\begin{array}{l}\text { Consommation } d^{\prime} \mathrm{O}_{2} \\
(\mathrm{mg} / 1000 \text { œufs } / \mathrm{h})\end{array}$ & $\begin{array}{l}\text { Nombre de } \\
\text { mesures }\end{array}$ \\
\hline $\begin{array}{l}2 \\
4 \\
10 \\
14 \\
25 \\
30 \\
40 \\
50 \\
65 \\
75 \\
\end{array}$ & $\begin{array}{l}2-4-8 \text { blastomères } \\
\text { morula } \\
\text { blastula } \\
\text { épibolie } \\
\text { fermeture blastopore } \\
\text { début de croissance de la vésicule } \\
\text { caudale } \\
\text { apparition du tube cardiaque } \\
\text { début mouvements du corps } \\
\text { pigmentation des yeux, pulsations } \\
\text { cardiaques } \\
\text { apparition d'éléments figurés dans } \\
\text { le plasma et formation du réseau } \\
\text { circulatoire } \\
\text { éclosion }\end{array}$ & $\begin{array}{l}0,10 \\
0,10 \\
0,10 \\
0,13 \\
0,13 \\
0,13 \\
0,13 \\
0,15 \\
0,17 \\
0,29 \\
0,54\end{array}$ & $\begin{array}{l}15 \\
20 \\
24 \\
28 \\
16 \\
\\
30 \\
35 \\
17 \\
\\
53 \\
\\
\\
99 \\
37\end{array}$ \\
\hline
\end{tabular}

Tableau 5 : Consommation d'oxygène par l'embryon de $C$. autumnalis migratorius à divers stades de développement (d'après CHERNYAEV, 1982).

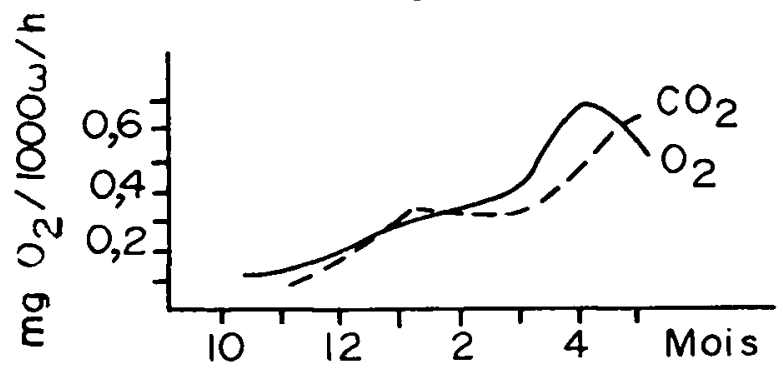

Figure 4: Evolution de la consommation d' $\mathrm{O}_{2}$ et l'excrétion de $\mathrm{CO}_{2}$ au cours de l'embryogenèse de $C$. autumnalis migratorius (en $\mathrm{mg}^{2} \mathrm{~d}^{\prime} \mathrm{O}_{2} / 1000$ coufs/h) (d'après CHERNYAEV, 1982).

\begin{tabular}{|l|l|l|l|l|l|}
\hline Oxygène dissous en mg/l & 12 & 4 & 3 & 2 & 1 \\
\hline Temps ł̇ l'éclosion en jours & 122 & 132 & 140 & 150 & 154 \\
\hline
\end{tabular}

Tableau 6 : Durée totale de la période d'incubation à $4^{\circ} \mathrm{C}$ suivant différentes concentrations en oxygène pour le Coregonus artedii (BROOKE et COLBY, 1980). 
Le développement de l'œuf est plus rapide pour une forte concentration en oxygène (tableau 6), de même que l'intervalle entre le premier et le dernier éclos est plus faible (tableau 7) (BROOKE et COLBY, 1980).

\begin{tabular}{|l|l|l|l|l|}
\hline Oxygène dissous en mg/l & 12 & 4 & 3 & 1 \\
\hline Intervalle en jours & 39 & 41,5 & 50 & 47 \\
\hline
\end{tabular}

Tableau 7: Etalement de la période d'éclosion a $4^{\circ} \mathrm{C}$ suivant différentes concentrations en oxygène pour le Coregonus artedii (BROOKE et COLBY, 1980.

\section{5) Sur les possibilités de retarder l'éclosion.}

La reproduction des corégones étant, en général, assez précoce (automne-hiver), les larves éclosent à une période où la richesse alimentaire du milieu est faible. En élevage, on recherche souvent à retarder l'éclosion et attendre que la quantité de plancton soit plus forte. La méthode la plus fréquemment utilisée consiste à pratiquer l'incubation à basse température. Nous savons que les embryons de quelques espèces de corégones supportent des températures voisines de $0^{\circ} \mathrm{C}$ (voir tableau 2). CHERNYAEV (1977) a signalé que des œu fs pouvaient se développer normalement à $-4^{\circ} \mathrm{C}$ ou dans la glace et se trouver en état de surfusion. FLUCHTER (1980) a signalé que des embryons de $C$. lavaretus incubés à $1^{\circ} \mathrm{C}$ voyaient leur éclosion différée de 8 semaines. $D^{\prime}$ autres essais faisant appel à des pressions élevées (jusqu'à 11 atmosphères) n'ont pas été couronnés de succès (MAECKLE, 1975).

\section{6) Traitement sanitaire devant l'incubation}

Il faut constamment enlever les œufs morts qui servent de support au développement des mycoses, lesquelles pouvant alors gagner les $œ u f s$ sains. On peut aussi traiter au vert de malachite ( 1 à $10 \mathrm{mg} / \mathrm{l}$ ) par bain de 30 minutes tous les 3 à 5 jours (STEFFENS. 1978).

\section{C - QUELQUES EXIGENCES PHYSIOLOGIQUES DES JEUNES STADES}

\section{1) Croissance}

La taille à l'éclosion des larves de Coregonus clupeaformis dépend de la durée de la période d'incubation, et non de la grosseur de l'ceuf mis en incubation (voir tableau 3). On a ainsi un alevin de $11,84 \mathrm{~mm}$ après 231 jours d'incubation et de $11,03 \mathrm{~mm}$ après 183 jours (BIDGOOD, 1974). Le poids à l'éclosion des alevins de Coregonus lavaretus est de $7,3 \mathrm{mg}$, pour une longueur de $11,7 \mathrm{~mm}$ (BOGDANOVA, 1980). En ce qui concerne le Coregonus species du Léman, il mesure à l'éclosion $10,7 \mathrm{~mm}$ et pèse environ $5 \mathrm{mg}$ (MACK, 1982). Le poisson est pleinement développé à $25 \mathrm{~mm}$ et $100 \mathrm{mg}$ (EINSELE, 1965).

Si on alimente les alevins de Coregonus fera avec des nauplius d'Artemia salina le premier jour après l'éclosion, on observe une légère décroissance du poids sec pendant 10-12 jours et ensuite une forte croissance (fig. 5). L'alevin atteint ainsi, après 20 jours, $16 \mathrm{~mm}$. Le taux de croissance spécifique du poids sec par jour allait de $-4 \%$ à $1-2$ jours à $6 \%$ à 30 jours et plus (fig. 6) (GUNKEL et KAUSCH, 1979).

L'alevin de Coregonus lavaretus, quant à lui, passe de $12,1 \mathrm{~mm}$ à $23,5 \mathrm{~mm}$ du $2^{\mathrm{e}}$ au $30^{\circ}$ jour, et de $8,3 \mathrm{mg}$ à $114 \mathrm{mg}$ pendant la même période, s'il est alimenté avec de la nourriture vivante (moina macroscopique et microcyclops sp.) à une température de $11,2^{\circ} \mathrm{C}$ (BOGDANOVA, 1980).

La croissance des alevins de Coregonus lavaretus en cage éclairée du coucher de soleil à minuit peut être décomposée en trois périodes : la première allant de fin mars à 


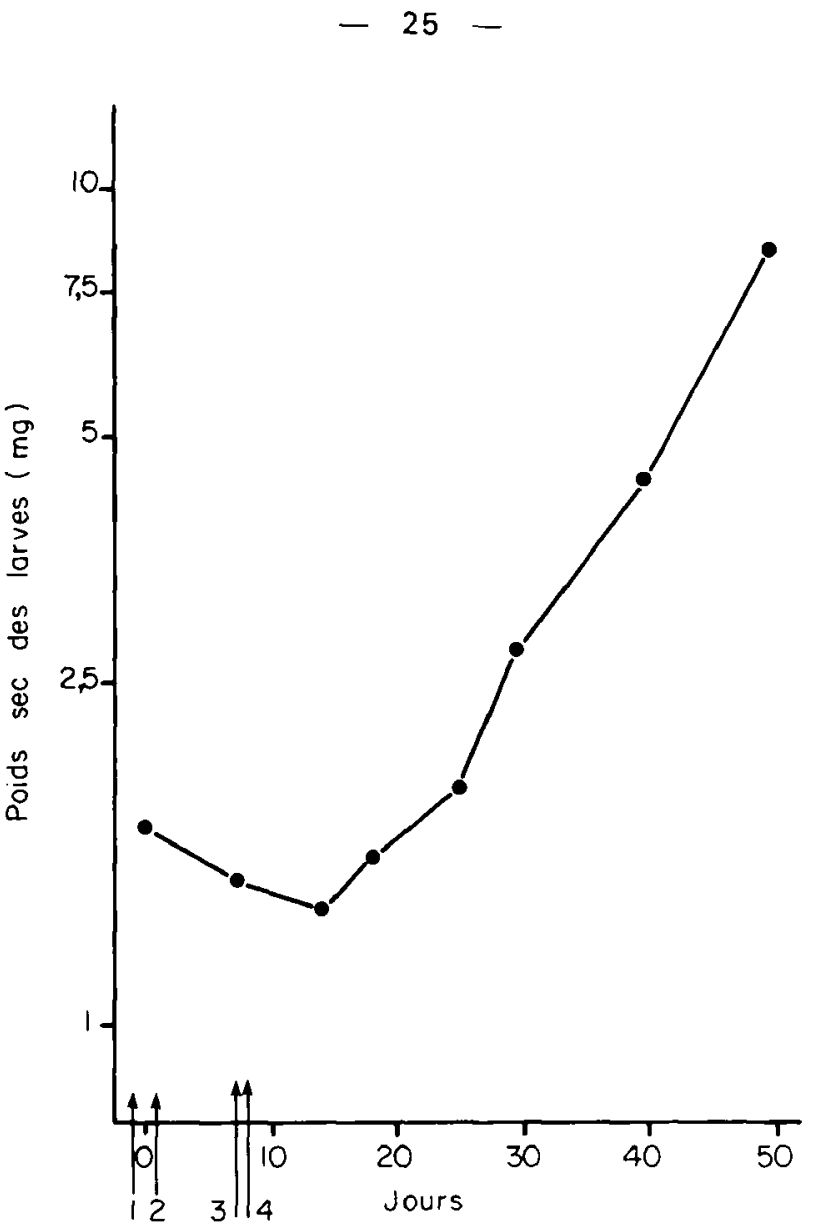

Figure 5 : Evolution du poids de la larve de Coregonus fera nourrie en excés avec des nauplius d'Artemia après éclosion. (GUNKEL et KAUSCH, 1979).

1. Éclosion

2. départ de la distribution avec des nauplius d'Artemia

3. dábut de prise de l'aliment

4. $50 \%$ des alevins s'alimentent.

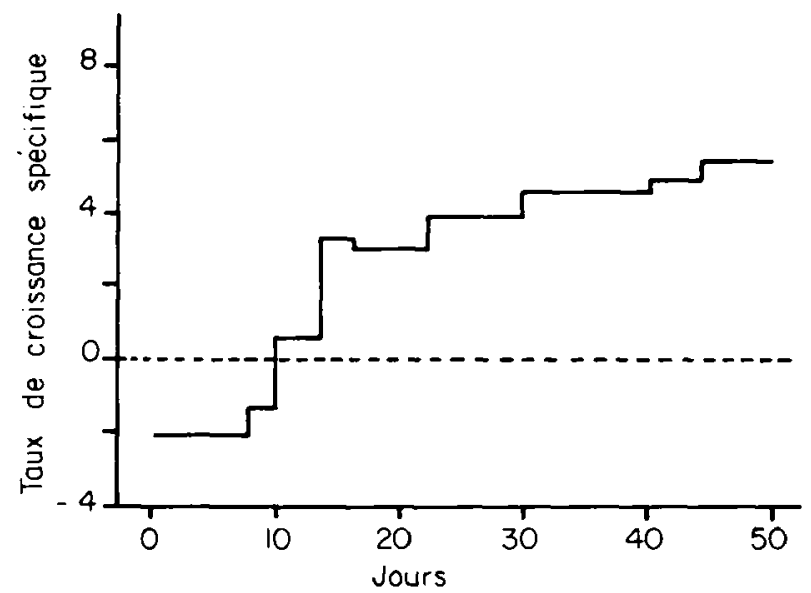

Figure 6: Relation entre le taux de croissance spécifique (exprimé en \% de variation du poids sec par jour) et le temps (jours) aprés éclosion. Le poids décroissant de l'alevin est exprimé comme taux de croissance négatif (GUNKEL ot KAUSCH, 1979). 
mi-mai où l'on obtient, dans les meilleurs cas, un gain de $27 \mathrm{mg} / \mathrm{j}$ et une croissance de $0,31 \mathrm{~mm} / \mathrm{j}$ pour une densité de 5140 à 16150 alevins en cage (cage de $8 \mathrm{~m}^{3}$ ); la deuxième, de mai à juillet, où l'on obtient dans les meilleurs cas, un gain de $47 \mathrm{mg} / \mathrm{j}$ et une croissance de $0,87 \mathrm{~mm} / \mathrm{j}$ pour une densité de 7000 alevins en cage. La dernière, d'août à mi-septembre où l'on arrive, dans les meilleurs cas, à un gain de $334 \mathrm{mg} / \mathrm{j}$ et une croissance de $1,1 \mathrm{~mm} / \mathrm{j}$ pour une densité de 3600 alevins par cage (résultats obtenus dans les lacs polonais). Le poids final individuel de l'alevin varie de 14 à $20 \mathrm{~g}$, les productions totales variant de 36 à $70 \mathrm{~kg}$ par cage, et le pourcentage de survie de 77,7 à 93.2 \% (BRYLINSKI et al., 1979)

\section{2) Alimentation}

La difficulté de la prise de nourriture par les larves est la principale raison de la mortalité larvaire. Parmi les facteurs importants, il faut citer la vitesse de nage des alevins en quête de nourriture ; elle est de $1,5 \mathrm{~cm} / \mathrm{sec}$. chez Coregonus c/upeaformis et peut atteindre 3,7 à $11,4 \mathrm{~cm} / \mathrm{sec}$. (HOAGMAN, 1973). Après éclosion, la vitesse est de l'ordre de 0,8 à $1,3 \mathrm{~cm} / \mathrm{sec}$. pour le Coregonus lavaretus et peut aller jusqu'à $2,5 \mathrm{~cm} / \mathrm{sec}$. en cas de fuite par exemple. Encore plus importante est la portée visuelle qui est de l'ordre de $10 \mathrm{~mm}$ pour un alevin de Coregonus lavaretus. Un jeune poisson de $5 \mathrm{~cm}$ de long peut se déplacer a $35 \mathrm{~cm} / \mathrm{sec}$. (FLUCHTER, 1980). On voit donc que la densité des proies est essentielle. RAISANEN et BEHMER (1982) pensent qu'en cas d'insuffisance de proies, des larves de Coregonus clupeaformis avalent des microbulles, ce qui entraîne des mortalités. Le même comportement a été signalé par JOHN et HASLER (1956), et FLUCHTER (1980) ci-après).

Mais la qualité de ces proies, taille en particulier, est aussi importante (voir

En été, l'alevin peut ingérer $40 \%$ du poids de son corps journellement, et même certains disent $90 \%$ avec du plancton naturel (FLUCHTER, 1980). GUNKEL et KAUSCH (1979) rapportent des augmentations de poids de plus de $100 \%$ du poids du corps humide par jour pour des alevins de 20 jours (fig. 7).

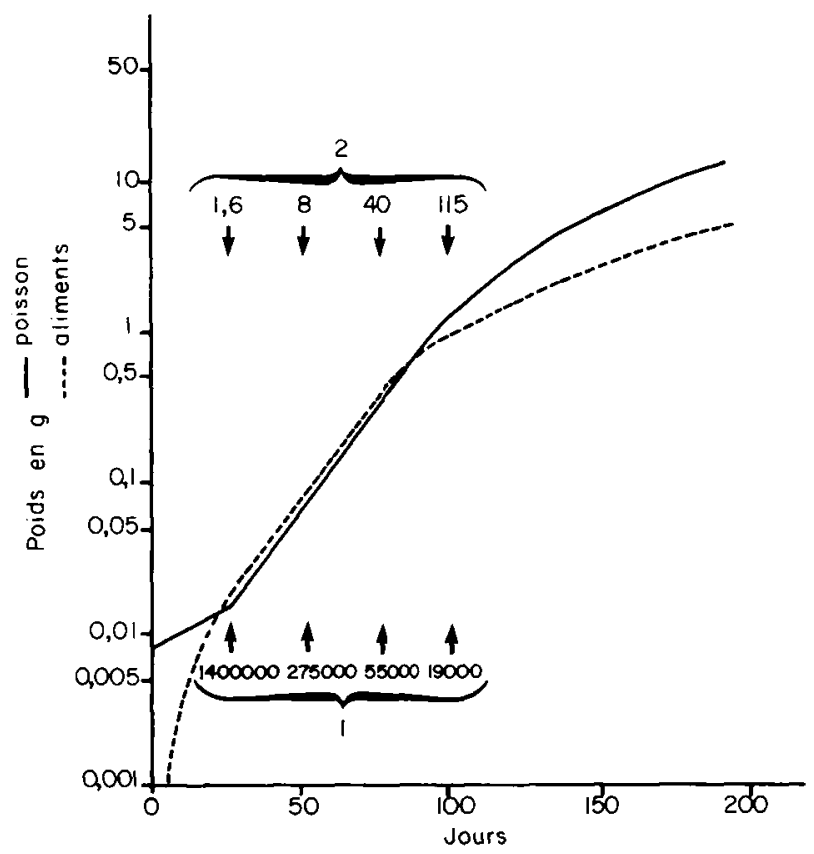

Figure 7 : Evolution des quantités d'aliments ingérés en g de poids humide/poisson/j et du poids des juvéniles au cours d'une période de 200 j postrésorption. (GUNKEL et KAUSCH, 1979).

1. mise en charge maxi - nombre de juvéniles/ha.

2. kg de zooplancton (poids humide) distribué par jour a 100000 juvéniles. 


\section{a) Début de l'alimentation}

Le passage à une nourriture exogène est une période critique pour l'alevin. En règle générale, c'est à ce moment que l'on observe la plus grosse mortalité quand la nourriture n'est pas disponible au bon moment, ou est inaccessible pour les larves. Les protozoaires, rotifères, moina, daphnies et autres formes de zooplancton n'excédant pas 0,5 à $0,6 \mathrm{~mm}$, doivent être donnés comme nourriture primaire (KOKOVA, 1978). D'ail. leurs, le diametre des formes de zooplancton doit être compris entre 50 et $300 \mu$ (STEFFENS. 1978). En effet, les alevins commencent à s'alimenter très tôt (de 5 à 7 heures après l'éclosion à 8 jours, selon les cas), à une taille de 9 à $11 \mathrm{~mm}$, et un poids de 3,5 a $5,6 \mathrm{mg}$. Il faut donc que la nourriture soit la plus petite possible (180 $\mu$ ) (tableau 8) (EINSELE, 1965). Les alevins les moins lourds à l'éclosion prennent leurs premières proies

\begin{tabular}{|c|c|c|c|c|c|c|}
\hline \multirow{3}{*}{$\begin{array}{l}\text { Taille de la } \\
\text { nourriture en } \\
\text { micromètre }\end{array}$} & \multicolumn{6}{|c|}{ Poids moyen des larves } \\
\hline & \multicolumn{2}{|c|}{$5,6 \mathrm{mg}$} & \multicolumn{2}{|c|}{$4,4 \mathrm{mg}$} & \multicolumn{2}{|c|}{$3.7 \mathrm{mg}$} \\
\hline & $\stackrel{\%}{\text { s'alimentant }}$ & $\begin{array}{l}\text { remplissage } \\
\text { de l'estomac }\end{array}$ & $\frac{\%}{\text { s'alimentant }}$ & $\begin{array}{l}\text { remplissage } \\
\text { de l'estomac }\end{array}$ & $\frac{\%}{\text { s'alimentant }}$ & $\begin{array}{l}\text { remplissage } \\
\text { de l'estomac }\end{array}$ \\
\hline $\begin{array}{l}470 \\
370 \\
250 \\
180\end{array}$ & $\begin{array}{r}15 \\
75 \\
100 \\
100\end{array}$ & $\begin{array}{l}10 \\
60 \\
75 \\
90\end{array}$ & $\begin{array}{r}0 \\
10 \\
45 \\
65\end{array}$ & $\begin{array}{r}5 \\
5 \\
25 \\
55\end{array}$ & $\begin{array}{r}0 \\
0 \\
40 \\
65\end{array}$ & $\begin{array}{r}- \\
20 \\
40\end{array}$ \\
\hline
\end{tabular}

Tableau 8 : Pourcentage de larves de corégone alimentées en relation avec la taille de l'organisme d'alimentation (EINSELE, 1965).

plus vite que les autres. Ainsi, à une température de $5^{\circ} \mathrm{C}$, un alevin qui pèse $3,7 \mathrm{mg} \mathrm{à}$ l'éclosion commence à s'alimenter le $12^{\circ}$ jour dans $50 \%$ des cas, tandis qu' un alevin de $5,6 \mathrm{mg}$ commencera à 33 jours (EINSELE, 1965). Le sac vitellin étant résorbé 3 à 5 jours après l'éclosion, certaines larves commencent à s'alimenter avant la résorption du sac vitellin (HUET, 1972). Le Coregonus fera prend de la nourriture à 5 jours, mais ne commence à la digérer qu'à 12 jours.

\section{b) Régime alimentaire}

Si l'on associe du plancton naturel et Artemia salina (phyllopode), les alevins ne mangent que Artemia dans un premier temps, puis, lor sque le poisson atteint $18 \mathrm{~mm}$ et un stade de développement caractérisé par la différenciation des nageoires (notamment nageoires dorsale et ventrale) et par le dépôt de guanine dans toute la cavité générale (métamorphose), il commence à manger des copépodes (FLUCHTER, 1980). Artemia est d'ailleurs une excellente nourriture d'élevage. II contient les substances nutritives essentielles pour les alevins de Corégone. On peut congeler Artemia, mais selon une méthode de congélation rapide. Si la congélation est lente, les substances attractives ne sont pas détruites, mais les substances essentielles le sont. Ainsi, dans ce cas, les alevins préfèrent Artemia aux copépodes, mais ils ne dépassent pas le stade de la métamorphose. Après congélation rapide, les substances essentielles ne sont pas détruites par les enzymes (FLUCHTER, 1980). Ces substances n'ont pas encore été identifiées.

Dans les 21 premiers jours, on obtient les meilleurs résultats sur Coregonus lavaretus, sur Coregonus peled et sur Coregonus autumnalis migratorius avec des proies de petite taille comme des protozoaires (paramécies) ou comme des rotifères ou des moinas. On obtient ainsi des taux de survie de 90 a $100 \%$ avec paramécies (densité faible de 17 alevins par litre) ou avec une nourriture mixte (paramécies, rotifères et moinas) (densité double), ou avec moina si la densité passe à 42 alevins par litre (fig. 8 et 9). II est donc approprié d'utiliser une nourriture mixte (paramécies et moinas, ou rotifères et moinas) pour des alevins après éclosion (KOKOVA, 1978). 


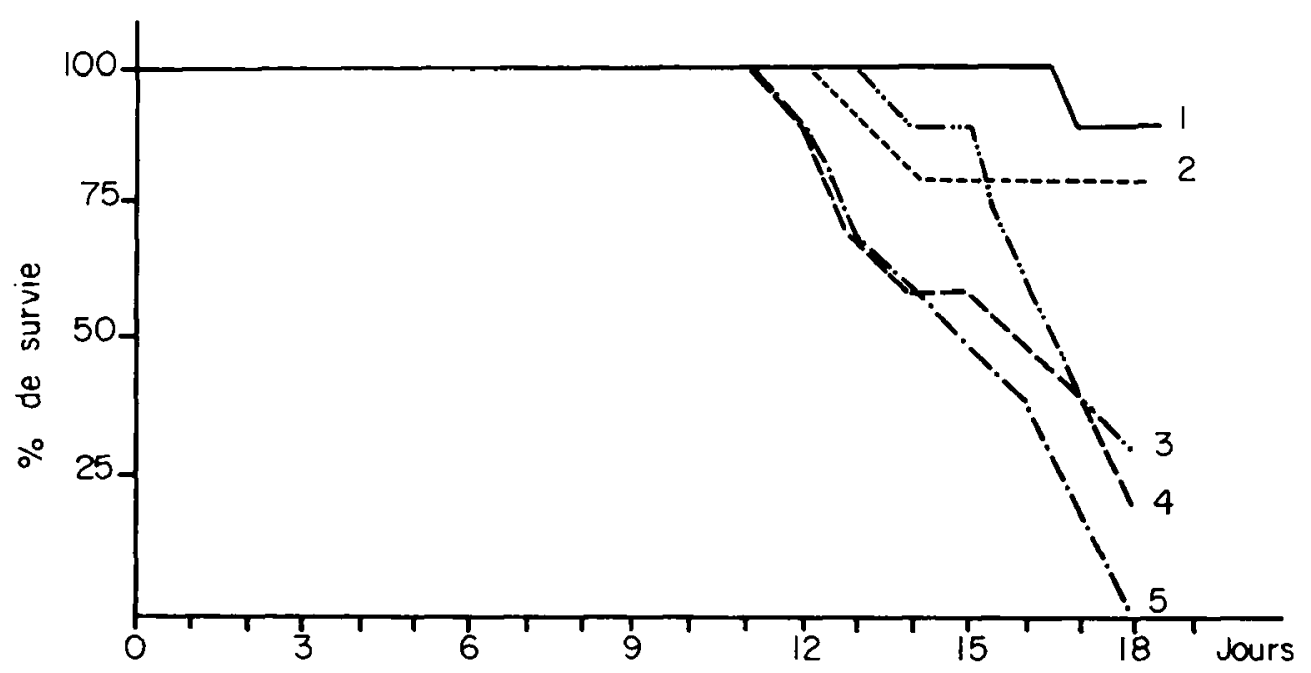

Figure 8 : Survie des larves de Coregonus lavaretus ludoga alimentées avec paramécie (1 et 2). paramécie sèche (3), daphnie (4) ou non alimentées (contróle 5) (densité : 17 larves par litre). (KOKOVA, 1978).

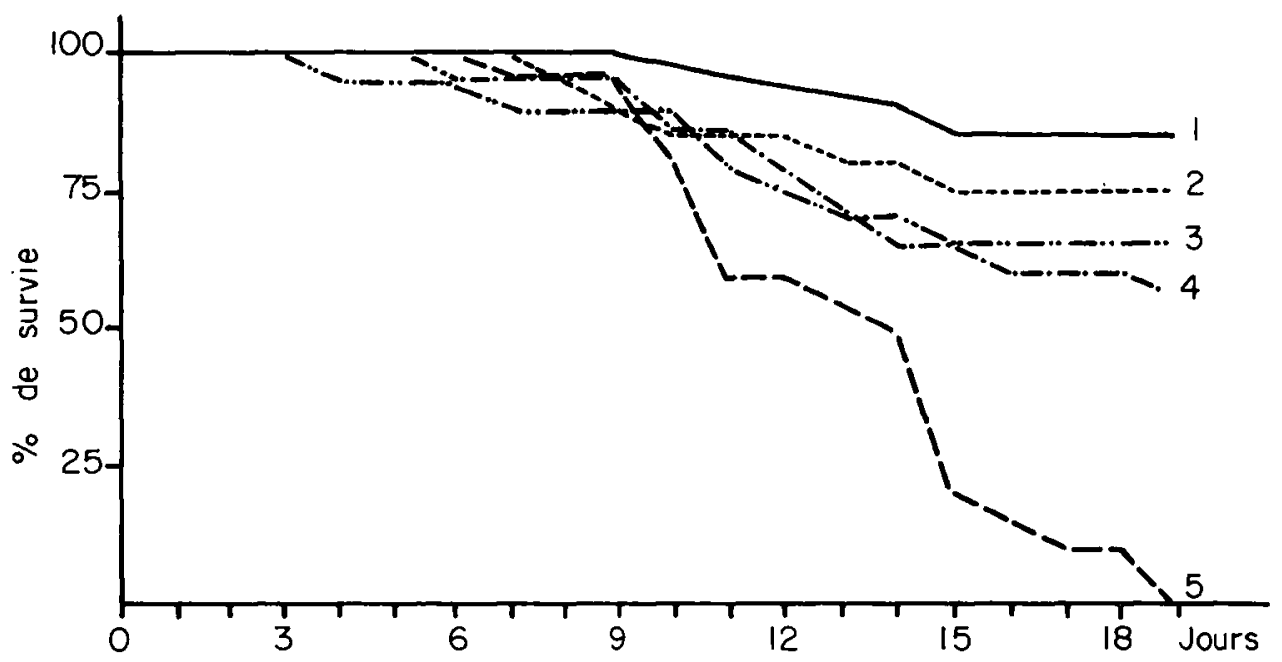

Figure 9 : Taux de survie des larves de Coregonus autumnalis migratorius nourries avec moina (1), rotifère et moina (2), paramécie et moina (3), paramétie, rotifère et moina (4) ou non alimentées (contróle 5) (densité : 42 larves par litre). (KOKOVA, 1978).

Les jeunes alevins, en présence de plancton, mangent surtout des copépodes, et quelquefois des rotifères, délaissant ainsi une grande partie du zooplancton qui peut, dans le cas d'élevage en bassins, se déposer au fond et entraîner des conséquences indésirables par les pollutions engendrées (HOCHMAN et al., 1978; FLUCHTER, 1980).

GRYGIEREK et al., (1980) donnent les chiffres suivants, pour l'alimentation du Coregonus albula, en présence de plancton naturel :

- pendant les 3 premiers jours : prise de rotifères seulement

- pendant les 9 jours suivants : $50 \%$ de rotifères.

$50 \%$ de crustacés

- ensuite jusqu'à 1 mois : $50 \%$ de crustacés.

$50 \%$ de larves d'insectes.

On peut voir sur la figure 10 un essai d'une représentation de l'atimentation de corégone pendant le premier mois. 


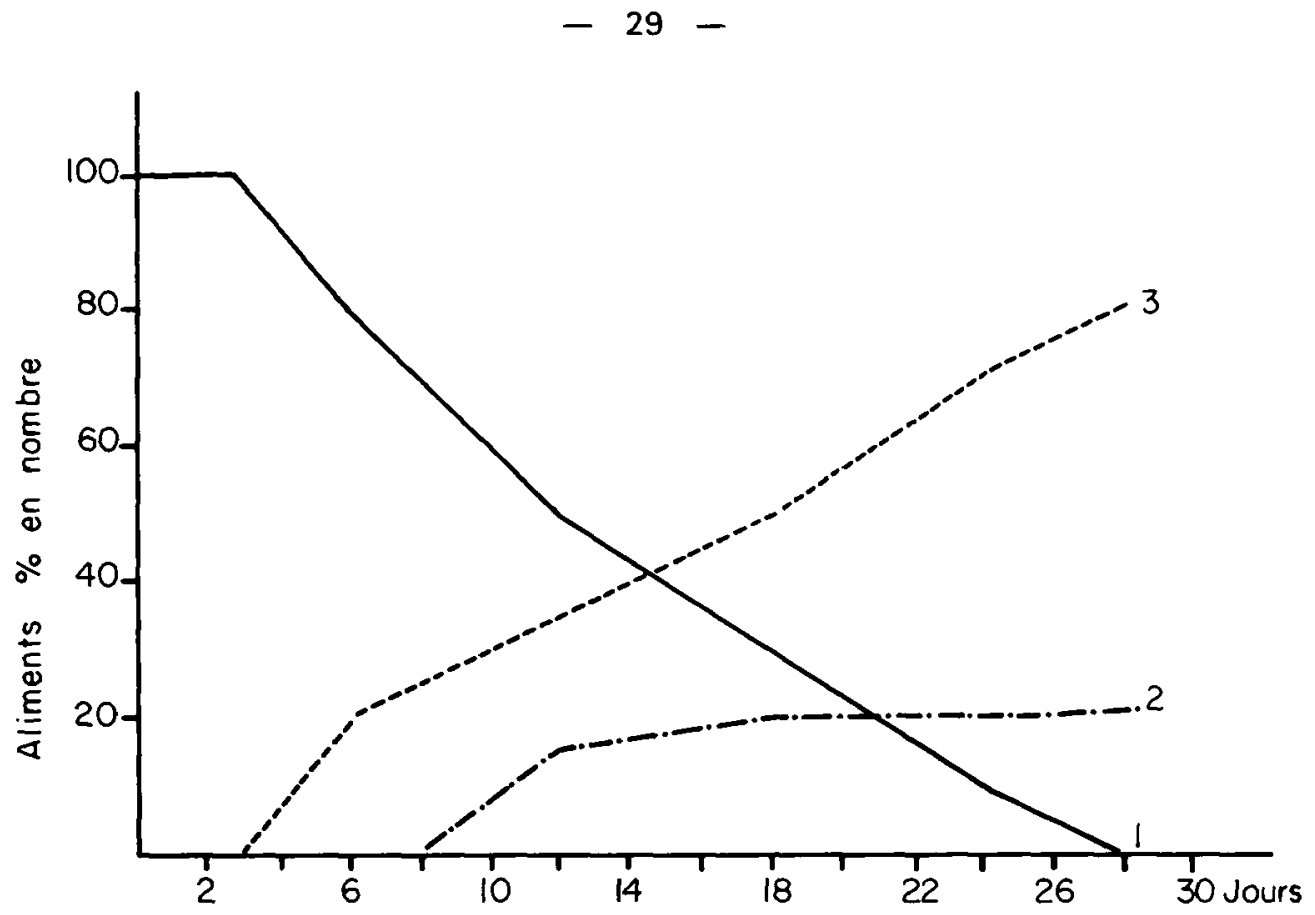

Figure 10 : Evolution du régime alimentaire du corégone pendant le mois qui suit la premiere prise de nourriture.

1 : petites formes (rotiféres, paramécies, moinas, etc.)

2 : larves d'insecte (chironomes, ephemeres, etc.)

3 : crustacés (d'après GRYGIEREK ot al., 1980).

Les alevins préfèrent ensuite le plancton crustacés (copépodes et cladocères), avec des spécimens individuels de benthos, comme les larves de chironomes, larves d'éphémères, etc. (Coregonus peled $\mathrm{Gm}$ ). Cependant, si le plancton crustacés manque, l'alevin consomme du necton (Corisca) et surtout du benthos qui peut représenter jusqu'à $100 \%$ de la ration. II y a donc un très haut niveau d'adaptation (HOCHMAN et al., 1978).II faut noter toutefois queMARCIAK (1979) identifie une grande différence, dans le spectre alimentaire des larves, entre Coregonus peled, Coregonus lavaretus et Coregonus albula, lorsqu'elles sont élevées en cage. Coregonus albula mange une nourriture très diversifiée (œufs de rotifères, daphnies sp., cyclops sp., cyanophycées, etc), Coregonus lavaretus choisit plutôt du plancton mobile et gros (cyclops sp. et daphnies sp.) et Coregonus peled des organismes très petits comme les œufs de rotifères.

En ce qui concerne les quantités consommées, on peut estimer que dans la première semaine un alevin mange 40 nauplius par jour, dans la deuxième semaine 80 et dans la troisième semaine 150 .

A 3-4 semaines on peut d'ailleurs donner du plancton de copépode non filtré. A $30 \mathrm{~mm}$, on compte 1000 formes de plancton par alevin et par jour (STEFFENS, 1978). BRAUM (1967, 1978) a estimé à 265 copépodes $/ 10 \mathrm{~h}$ à $15,6^{\circ} \mathrm{C}$ la consommation de juvéniles de $C$. wartmanni de 31 jours.

GUNKEL et KAUSCH (1979) ont évalué la quantité de $\mathrm{kg}$ de zooplancton à distribuer par jour pour nourrir 100000 juvéniles de Coregonus fera :

$-1,6 \mathrm{~kg}$ à 25 jours $(16 \mathrm{mg} ; 1.7 \mathrm{~cm})$

$-8 \mathrm{~kg}$ a 50 jours $(65 \mathrm{mg} ; 2.5 \mathrm{~cm})$

- $40 \mathrm{~kg}$ à 75 jours $(0.4 \mathrm{~g} ; 4 \mathrm{~cm})$

$-115 \mathrm{~kg}$ à 100 jours $(1,6 \mathrm{~g}: 6,5 \mathrm{~cm})$.

\section{3) Influence de la température}

L'alevin se nourrit entre 2 et $26^{\circ} \mathrm{C}$. A une température de $10^{\circ} \mathrm{C}$ la nourriture est prise une fois par jour, tandis qu'a $18^{\circ} \mathrm{C}$ la digestion se fait seulement en 4 heures (FLUCHTER, 1980). On obtient sur Coregonus lavaretus alimenté pendant 30 jours avec 
de la nourriture vivante (moina macroscopique et microcyclops sp.) une différence de $3,8 \mathrm{~mm}$ et de $66 \mathrm{mg}$ lorsque l'on passe de $9,6^{\circ} \mathrm{C}$ à $11,2^{\circ} \mathrm{C}$ (BOGDANOVA, 1980).

Sur les 28 premiers jours, dans des conditions optimales, le taux de croissance de Coregonus artedii augmente de façon significative de 3 à $18^{\circ} \mathrm{C}$ et le taux de mortalité est bas de $3^{\circ}$ a $13^{\circ}$ (voir fig. 11 ).

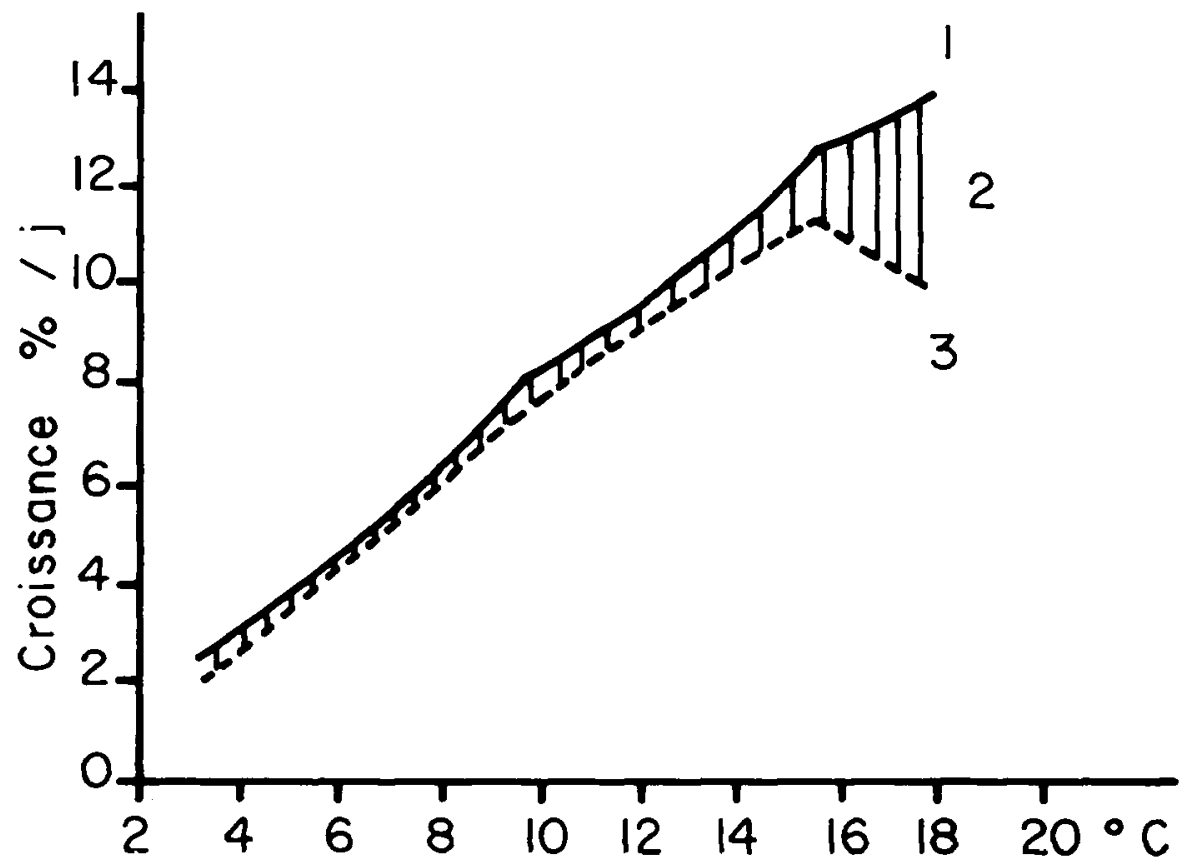

Figure 11 : Effet de la température sur le taux de croissance instantan $\measuredangle$ et sur la mortalité des larves de Coregonus artedii pendant les 28 premiers jours après éclosion. (McCORNICK, 1971).

1 : croissance - 2 : mortalités - 3 : gain net de biomasse.

Les températures optimales se situent entre $13^{\circ}$ et $18^{\circ} \mathrm{C}$, car le fort taux de croissance compense l'augmentation de la mortalité (McCORNICK et al., 1971 ; STEFFENS, 1978)

La température létale pour une larve de Coregonus clupeaformis acclimatée à $5^{\circ} \mathrm{C}$ est de $20,6^{\circ} \mathrm{C}$ et de $26,6^{\circ} \mathrm{C}$ lorsqu'elle s'est acclimatée à $22,5^{\circ} \mathrm{C}$ (EDSALL et ROTTIER, 1976). Des alevins de Coregonus clupeaformis de $0,14 \mathrm{~g}$ sont acclimatés à des températures de $24,5,25$ et $28^{\circ} \mathrm{C}$ pendant 1 minute pour être remis à leur température d'acclimatation en compagnie de perches (Perca flavescens) d'un an. Un nombre significativement plus élevé de corégones a été capturé par unité d'attaque (données combinées des poissons soumis et non soumis au choc) à 15 et $18^{\circ} \mathrm{C}$ jusqu'à $10^{\circ} \mathrm{C}$. Les alevins soumis au choc sont nettement plus vulnérables à la capture par les perches que les témoins non soumis au choc (YOCOM et EDSALL, 1974).

\section{4) Le rôle de l'oxygène}

L'expérience retracée sur la figure 12 a été effectuée sur des larves qui pesaient $5,6 \mathrm{mg}$ à l'éclosion. On peut ainsi voir que la mortalité atteint $100 \%$ si la concentration en oxygène est inférieure à $0.9 \mathrm{mg} / \mathrm{l}$ et que l'on obtient une mortalité inférieure à $10 \%$ pour une concentration en oxygène supérieure à $7 \mathrm{mg} / \mathrm{I}$. On remarque également que la demande en oxygène est influencée par la température.

D'autre part, une larve plus petite résiste moins bien à la privation d'oxygène. $50 \%$ des alevins de $3,7 \mathrm{mg}$ meurent à une concentration de $4.9 \mathrm{mg} / \mathrm{I}$ d'oxygène à $11.5^{\circ} \mathrm{C}$ : alors qu'il faut descendre à une concentration de $3,7 \mathrm{mg} / 1$ pour un alevin de $5,6 \mathrm{mg}$ (EINSELE, 1965). Lors de l'élevage, la teneur en oxygène dissous dans l'eau doit toujours être au-dessus de $5 \mathrm{mg} / \mathrm{I}$, et même. si possible, au-dessus de 8 à $9 \mathrm{mg} / \mathrm{I}$. 


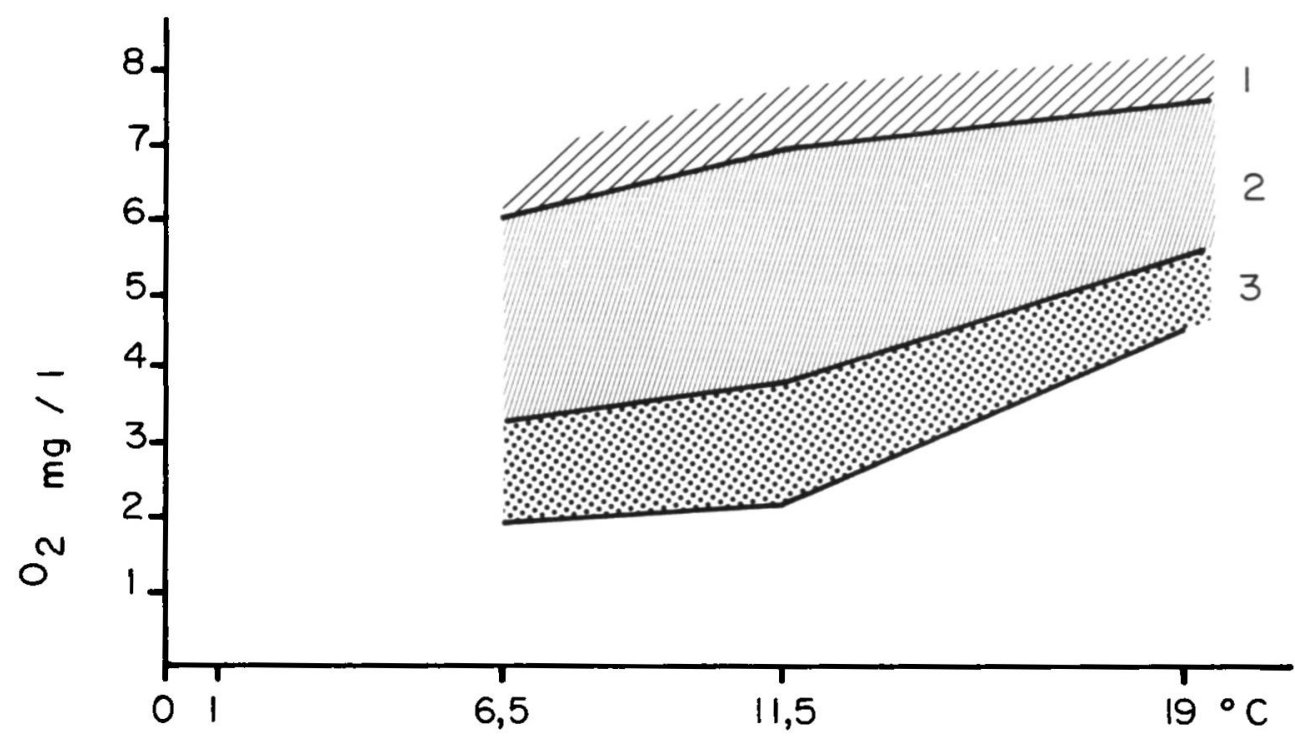

Figure 12 : Demande en oxygène des larves de corégone en fonction de la température, d'après EINSELE (1965). Evolution du taux de mortalité : $1:<10 \%-2$ : entre 10 et $50 \%-3$ : entre 50 et $100 \%$

\section{5) Influence de l'éclairement sur la prise de la nourriture}

L'alevin ne prend de la nourriture que si l'intensité lumineuse est supérieure à 0.1 lux (FLUCHTER, 1980). Elle est par exemple possible au niveau de l'éclairage lunaire (HOAGMAN, 1973). II faut normalement une intensité lumineuse supérieure à 100 lux pour que les alevins se nourrissent convenablement (EINSELE, 1965). Une intensité de 20000 lux stimule l'activité de recherche (FLUCHTER, 1980). Dans un gradient de lumière de 1 à 2475 lux, il n'y a pas de concentration de larves (HOAGMAN, 1973), ce qui autorise l'élevage en cage illuminée.

\section{D - Sur quelques teChniques d'Elevage des Jeunes stades}

\section{1) Elevage en bassin avec aliments inertes}

Nous venons de voir que les larves de corégones pouvaient consommer des proies vivantes en captivité et qu'il était ainsi possible de procéder à un élevage. De nombreux auteurs ont tenté de réaliser un tel élevage en apportant des proies inertes avec des succès divers.

On remarque, sur la figure 8 , que les larves nourries avec des daphnies et des paramécies sèches meurent et suivent, à peu près, l'évolution de la courbe témoin sans nourriture. Ces alevins restent donc affamés. Ceci est sans doute dû, pour les daphnies, à leur grosseur (elles restent dans la première section de l'intestin et les larves meurent). Les alevins ayant mangé de petites daphnies survivent. Pour les paramécies sèches, elles tombent au fond au bout d'un certain temps et elles se trouvent inaccessibles pour l'alevin (KOKOVA, 1978).

RAISANEN et BEHMER (1 982) observent aussi que du zooplancton (surtout constitué de Diaptomus) distribué à l'état congelé n'est plus consommé par des larves de Coregonus clupeaformis dès qu'il s'est sédimenté sur le fond. Cependant, s'il est remis en suspension à l'aide de jets d'eau intermittents, il est à nouveau consommé par les larves. Pour permettre une prise immédiate avant sédimentation, MEDGYESY et WIESER (1982) ont réalisé un appareil de distribution automatique du plancton congelé qui permet, d'autre part, d'éviter une détérioration de la qualité du plancton et la fuite de nutriments essentiels qui semble intervenir rapidement après décongélation (voir ci-dessus) 
On note également que les œufs de crevettes d'eau salée constituent une bonne nourriture pour le Coregonus artedii à une température de $5,2^{\circ} \mathrm{C}$ (HALE, 1970). Mais avec une nourriture artificielle de truite, les alevins de Coregonus lavaretus ne survivent pas au-delà de 30 jours à $11,2^{\circ} \mathrm{C}$ ou à $9,6^{\circ} \mathrm{C}$, qu'elle soit donnée au début de l'alimentation, ou au bout du treizième jour. La nourriture vivante, témoin avec des moinas macroscopiques et de microcyclops sp., donne, par contre, des résultats favorables (BOGDANOVA, 1980).

RAISANEN et BEHMER (1982) observent aussi que les juvéniles de Coregonus clupeaformis n'acceptent des granulés qu'au-delà de 13 semaines d'âge et de $40 \mathrm{~mm}$ de longueur.

\section{2) Technique d'élevage en cage}

Généralement, on utilise des cages immergées de $8 \mathrm{~m}^{3}(2 \times 2 \times 2 \mathrm{~m})$ (fig. 13 et 14) (BATURO, 1979 ; WOLF, 1979 ; ANWAND, 1976 ; JAGER et NELLEN, 1980 et 1981 ; BRYLINSKI et al., 1979 ; URYN, 1979). Ces cages sont plongées, suivant le cas, de $2 \mathrm{~m}$ (WOLF, 1979) à 6-8 m (ANWAND, 1976 ; JAGER et NELLEN, 1980).

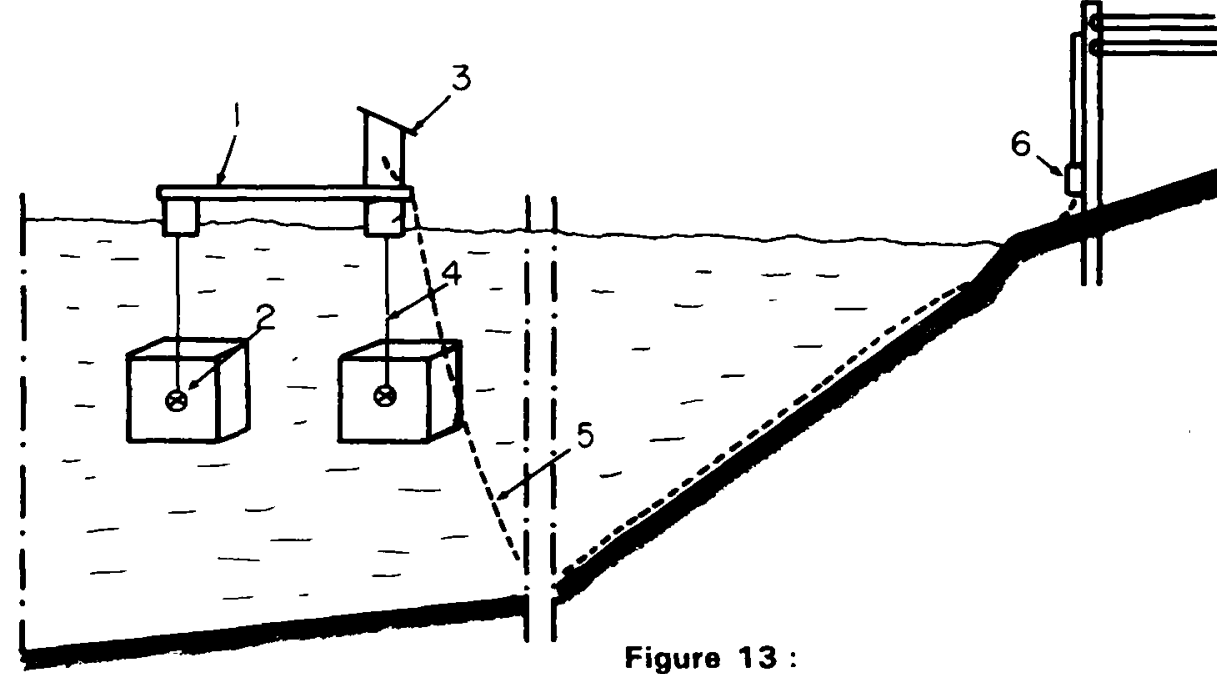

Figure 13:

Schéma d'implantation de cages en lacs.

1. ponton flottant

2. cage avec une lampe électrique

3. transformateur

4. câble supportant la cage

5. câble blectrique

6. contrôle automatique de l'allumage.

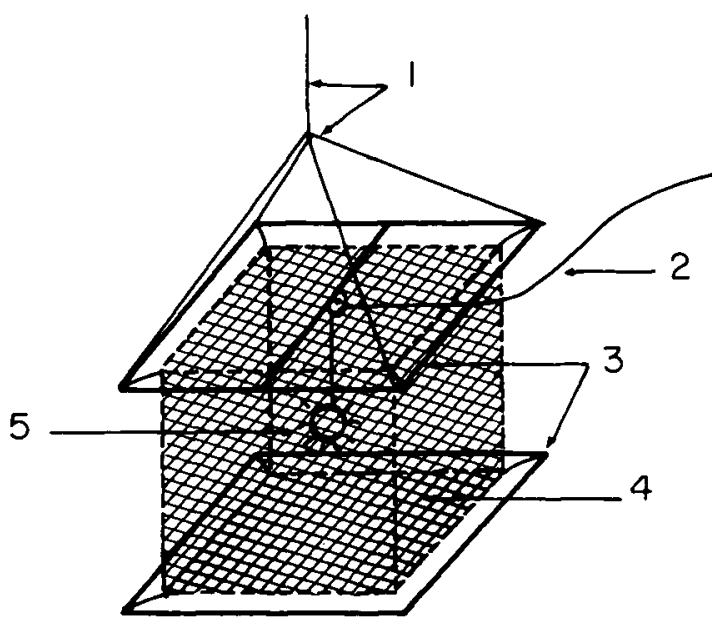

Figure 14 :

Détail d'une cage immergée (URYN, 1979)

1 : câble supportant la cage

2 : cáble électrique

3 : cadre métallique

4 : filet de la cage

5 : lampe électrique 
JAGER et NELLEN (1981) définissent la profondeur à 2 fois la lecture du disque de Secchi ; la cage se trouve ainsi placée dans une zone où le développement du fouling est le plus réduit.

Elle possède généralement une ou plusieurs lampes pour attirer le plancton dans la cage (voir fig. 11 et 12). L'éclairage est constitué soit de 3 ampoules de 60 W (JAGER et NELLEN, 1980 ; ANWAND, 1976), soit d'une ampoule de $100 \mathrm{~W}$ (JAGER et NELLEN, 1981 ; WOLF, 1979). Cet éclairage sert à attirer le plancton (crustacés) dans la cage. On augmente ainsi la densité du plancton de 48 fois (KOZIANOWSKI, 1979 ; ANWAND. 1976 : STEFFENS, 1978). Nous observons d'ailleurs, sur le tableau $n^{\circ} 9$, que la croissance en poids et le taux de survie sont bien meilleurs lorsque la cage est éclairée (URYN, 1979).

\begin{tabular}{|c|c|c|c|c|}
\hline \multirow{2}{*}{$\begin{array}{c}\text { Empoissonnement } \\
\text { densité (par } \mathrm{m}^{3} \text { ) }\end{array}$} & \multicolumn{3}{|c|}{ Résultats } \\
\cline { 2 - 5 } & $\begin{array}{c}\text { Poids moyen individuel (mg) } \\
\text { illuminée }\end{array}$ & $\begin{array}{c}\text { cage non } \\
\text { illuminée }\end{array}$ & $\begin{array}{c}\text { cage } \\
\text { illuminée }\end{array}$ & $\begin{array}{c}\text { cage non } \\
\text { illuminée }\end{array}$ \\
\cline { 2 - 5 } & & 63.9 & 73,68 & 40,38 \\
$\begin{array}{c}\text { au } 30 \text { mars de survie (\%) } \\
6655 \\
304\end{array}$ & 130.5 & 719 & 81,88 & 37,42 \\
325 & 2385 & 3791 & 82,54 & 40,98 \\
\hline $\begin{array}{c}\text { au } 22 \text { mai } \\
325\end{array}$ & 10978 & & & \\
\hline
\end{tabular}

Tableau 9 : Performances de croissance et de survie sur un élevage d'alevins de Coregonus lavaretus (L.) en cage illuminée ou non. (URYN, 1979).

L'élevage se passe, généralement, en 3 périodes qui demandent des filets de maille différente, ceci dans le cas de juvéniles élevés jusqu'à la fin de l'été (10 à $20 \mathrm{~g}$ ). Au début, pendant 4 à 6 semaines, on peut utiliser des mailles de 0.3 à $0.8 \mathrm{~mm}$ puis, pendant 6 à 8 semaines, des mailles de 0.8 à $2 \mathrm{~mm}$, et enfin des mailles de 5 à $5.6 \mathrm{~mm}$ jusqu'à la fin de l'élevage (JAGER et NELLEN, 1980 ; BRYLINSKI et al., 1979 ; A.NWAND, 1976 ; URYN, 1979).

Mais il faut nettoyer souvent les cages, car les algues ont tendance à obstruer les mailles et à empêcher ainsi le plancton de rentrer dans la cage, surtout lorsque la température de l'eau dépasse $10^{\circ} \mathrm{C}$ (WDLF, 1979).

\section{3) Technique d'élevage en étang}

a) La hauteur d'eau dans l'étang

Elle est de 0,7 à $1 \mathrm{~m}$, selon GRYGIEREK et al. (1980).

\section{b) Mise en eau}

Le déversement des larves dans l'étang intervient 1.5 à 2 semaines après la mise en eau si la température de l'eau est supérieure à $15^{\circ} \mathrm{C}$, et 4 a 6 semaines entre 10 et $15^{\circ} \mathrm{C}$. Mais si la température de l'eau est inférieure à $10^{\circ} \mathrm{C}$, l'étang doit être mis en eau avant l'hiver (6 a 7 mois avant le déversement). La biocénose se trouve ainsi, lors du déversement des larves, à un stade plus avancé que si la mise en eau a lieu au printemps (GRYGIEREK et al., 1980).

\section{c) Fertilisation}

On peut mettre, à la mise en eau, $2 \mathrm{~T} / \mathrm{ha}$ d'engrais organique (engrais vert, 
compost, fumier, etc.), plus $5 \mathrm{mg}$ d'azote par litre (urée, nitrates, etc.) et $1 \mathrm{mg}$ de $\mathrm{P}_{2} \mathrm{O}_{5} / \mathrm{I}$ (superphosphate) ou bien $2 \mathrm{~T}$ de lisier par ha à la place des engrais minéraux. Si la mise en eau s'effectue avant l'hiver on peut mettre plus d'engrais organique et moins d'engrais minéral (GRYGIEREK et al., 1980).

\section{d) Importance de la prédation}

Des juvéniles un peu plus gros, ou des adultes, peuvent manger des alevins mais il n'y a pas de preuves décisives. II peut y avoir une prédation par les perches (FLUCHTER, 1980). Lors d'un déversement d'alevins de Coregonus autumnalis migratorius, dans le Posolskiy lagon, il y a eu une forte prédation par les perches, gardons et ides de 1 à 2 ans. Une seule perche d' 1 an consomme 30 alevins de Coregonus autumnalis migratorius par jour, un gardon 26 et un ide 74 (YELL'TSOVA, 1976). Il y a donc lieu de se débarasser des prédateurs avant la mise en eau.

\section{e) Pathologie}

Dans le cas de l'élevage en bassin, RAISANEN et BEHMER (1982) signalent des problèmes de maladies branchiales entre 4 et 10 semaines, entraînant des mortalités importantes. Les branchies, couvertes de mucus, ne présentaient pas de bactéries pathogènes. Plus tard, vers 18 semaines d'élevage, ces mêmes auteurs observent des signes d'hémorragies externes et des pourritures de nageoires qu'ils ont traitées par des bains salés et de la terramycine introduite dans l'aliment.

Dans le cas d'un élevage en cage, les affections parasitaires sont considérées comme limitées (12 espèces de parasites trouvées chez Coregonus pe/ed). La première année de l'élevage on observe quelques dommages pathologiques dus à l'apparition de Branchiomyces sp. La deuxième année, de petites lésions sont apparues sur les branchies pendant l'invasion de $E$. siebaldi, de 1 . multifibris et de $C$. hirudinello. La comparaison des poissons infectés et non infectés montre que la croissance et le poids n'ont pas été affectés par ces parasites. L'alevin de Coregonus peled était dans de très bonnes conditions et montrait une haute survie (BATURO, 1979).

\section{E - CROISSANCE DE L'ADULTE ET REPRODUCTION}

\section{1) Croissance}

La croissance du grand corégone (Coregonus clupeaformis) est limitée à une brève période de l'année dans les lacs Hélène, Nathalie et Ronge du Canada. On remarque, en effet, sur la figure 15, qu'elle ne dure que 2 mois (juillet et août). La croissance est, de ce

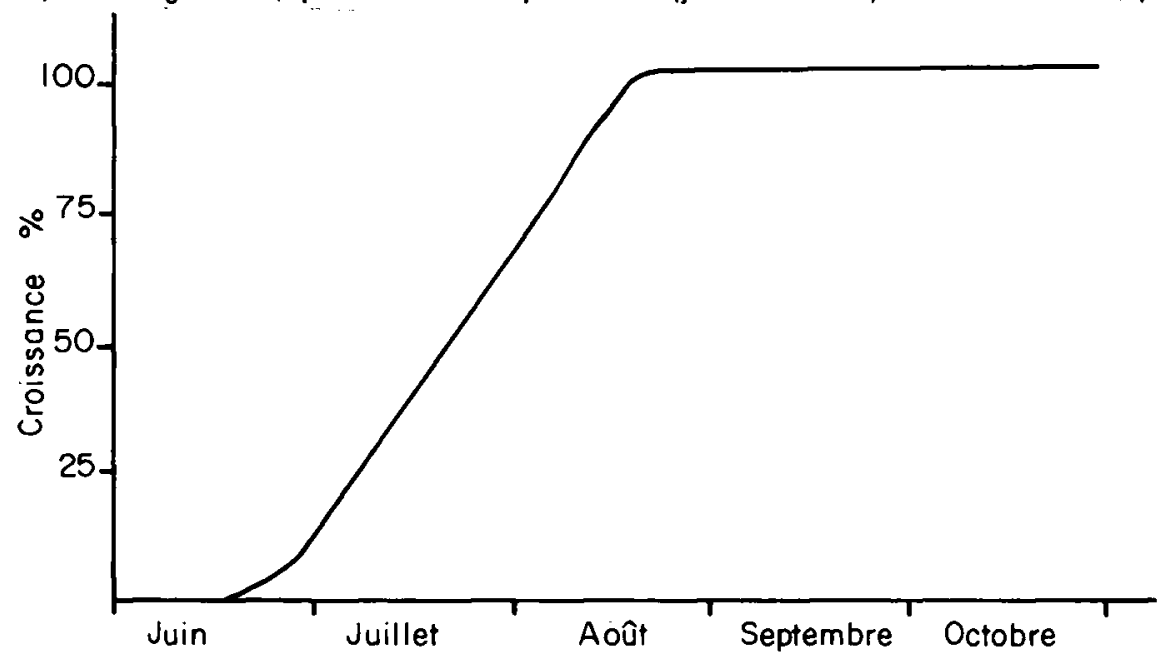

Figure 15 : Croissance saisonnière exprimbe en pourcentage de l'accroissement annuel pour l'ensemble des corégones de 4-9 ans capturés dans le lac Nathalie, (DUMONT et FORTIN, 1978). 
fait, assez lente (QUADRI, 1968 ; DUMONT et FORTIN, 1978), mais elle dépend aussi de la température. Ainsi, on peut voir, sur la figure 16, que le poids de Coregonus peled $\mathrm{Gm}$. à un âge de 3 ans, augmente avec la température de l'air en été (mois de pleine croissance) (KANEP, 1973).

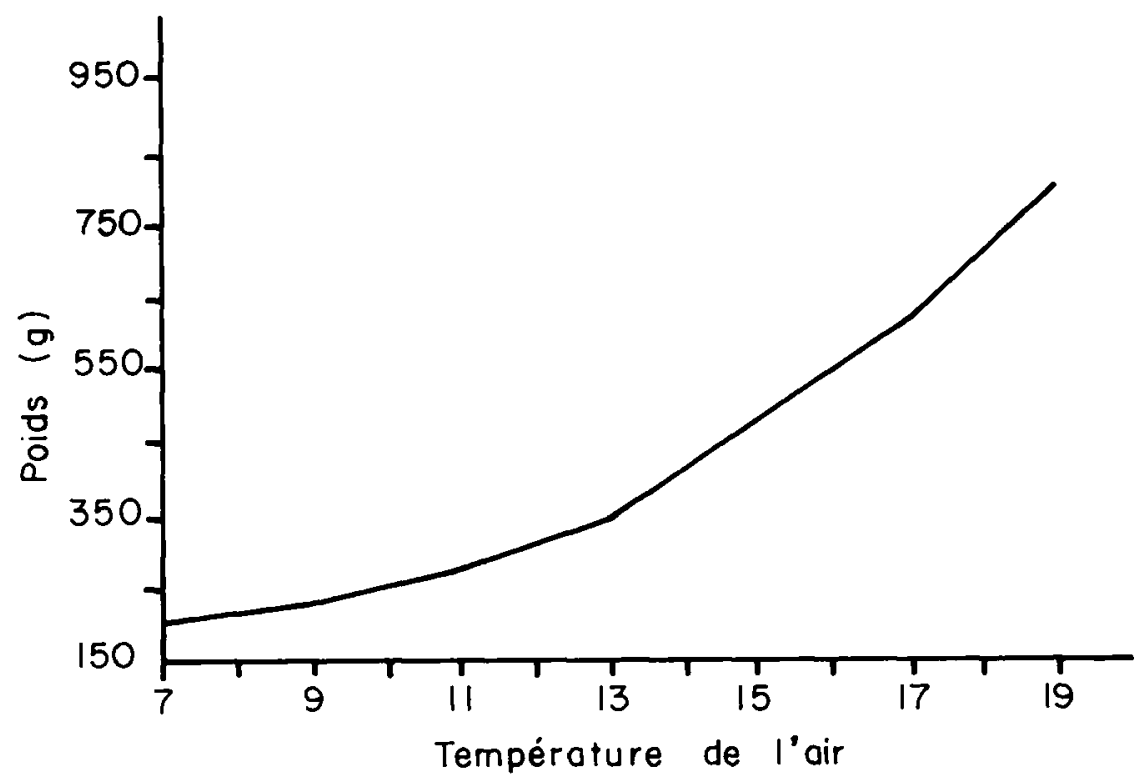

Figure 16 : Relation entre le poids moyen du Coregonus peled (Gm) et la temperature de l'air pendant les mois de juin à août, à l'âge de 3 ans. (KANEP. 1973).

Les performances de croissance sont bonnes entre 13 et $18^{\circ} \mathrm{C}$ chez l'adulte comme chez l'alevin (paragraphe C.1). Toutefois, une croissance existe, mais est assez faible, pour des températures inférieures à $10^{\circ} \mathrm{C}$ en été. On note ainsi une augmentation de croissance dans les régions plus chaudes, mais, en contrepartie, la durée de vie semble diminuer (KANEP, 1973 ; RESHETNIKOV, 1966).

\section{a) Croissance en longueur}

Elle diffère beaucoup suivant les lacs. Mais elle est toujours plus forte les premières années de la vie. Ainsi, dans les lacs Hélène et Nathalie, on obtient une croissance de $64 \mathrm{~mm}$ la première année qui ensuite diminue et vient s'annuler vers 13 . 14 ans $(450 \mathrm{~mm}$ ) (Coregonus clupeaformis) (DUMONT et FORTIN. 1978) (fig. 17).

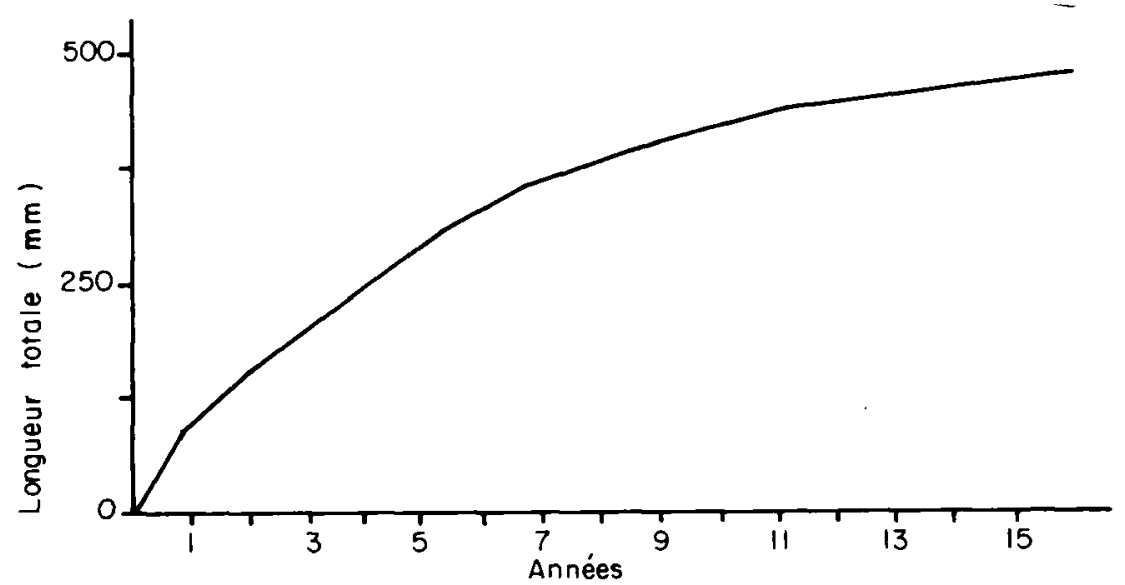

Figure 17 : Croissance en longueur de Coregonus clupeaformis du lac Hélène. (DUMONT et FORTIN. 1978). 
On obtient des résultats similaires dans le Chunozero (RESHETNIKOV, 1966). Par contre, si l'on prend Coregonus peled dans le lac Titovo, on constate une croissance rapide : $4.9 \mathrm{~cm}$ le 17 juillet de l'année d'éclosion, $32 \mathrm{~cm}$ le 15 octobre de l'année suivante (VOLGIN, 1973).

\section{b) Croissance en poids}

Contrairement à la croissance en longueur, la croissance en poids augmente jusqu'à un certain àge, puis diminue ensuite. Nous avons ainsi pour le Coregonus clupeaformis des lacs Hélène et Nathalie une croissance de 20 à $25 \mathrm{~g}$ la première année, de $100 \mathrm{~g}$ la septième année (qui est le maximum de la croissance ici) et de $50 \mathrm{a} 60 \mathrm{~g}$ vers 14-15 ans (fig. 18). Les poissons pèsent entre 850 et $1000 \mathrm{~g}$ au bout de 14 ans

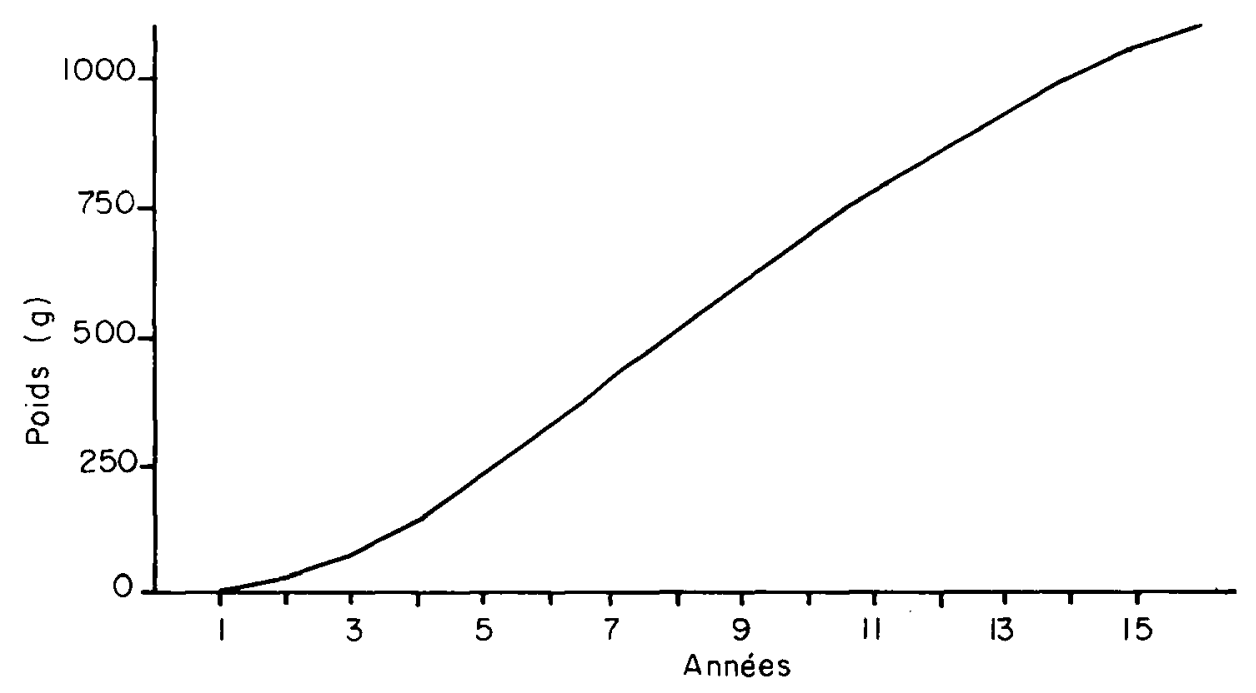

Figure 18 : Croissance en poids de Coregonus clupeaformis du lac Hélène. (DUMONT et FORTIN, 1978).

(DUMONT et FOF IIN, 1978). Dans le cas d'une croissance plus rapide comme chez Coregonus peled du lac Titovo, on observe un poids de $2,1 \mathrm{~g} \mathrm{le} 17$ juillet de l'année d'éclosion et de $559 \mathrm{~g}$ le 15 octobre de l'année suivante (VOLGIN, 1973). Le poids adulte du lavaret se situe, lui, entre 1 et $3 \mathrm{~kg}$ (HUET, 1972).

\section{2) Régime alimentaire}

Coregonus albula et Coregonus peled sont zooplanctonophages. Les lavarets, au sens large, sont zooplanctonophages ou benthophages ou changent de nourriture suivant les circonstances. Ils exigent, tous, des lacs riches en zooplancton (KOSIKOWSKA, 1976).

Le grand corégone (Coregonus clupeaformis) se nourrit de crustacés et d'hydracariens, mais mange également beaucoup d'insectes adultes et d'exuvies, de larves et pupes d'insectes aquatiques et quelquefois de mollusques. Il peut manger jusqu'à $100 \%$ de la ration en poisson (Gasterosteus aculeatus) lorsque les autres nourritures font défaut. II s'agit d'un poisson qui s'adapte facilement à l'alimentation disponible (DUMONT et FORTIN, 1978) et dont le spectre des proies consommées augmente au cours de la première année d'âge (RECKAHN, 1970). Le Coregonus muksun (Pallas) d'eau douce se nourrit, lui, d'insectes (chironomes) et essentiellement de crustacés (ostracodes et copépodes-cyclops-) et également de calanoïdes (ALEKSANDROVA, 1973).

Si l'alimentation change suivant la période de l'année chez le Coregonus albula (fig. 19), elle varie également suivant l'heure. En effet, le Coregonus albula migre pendant la journée de la surface (le soir) vers le fond (le matin). II trouve, par exemple, le 


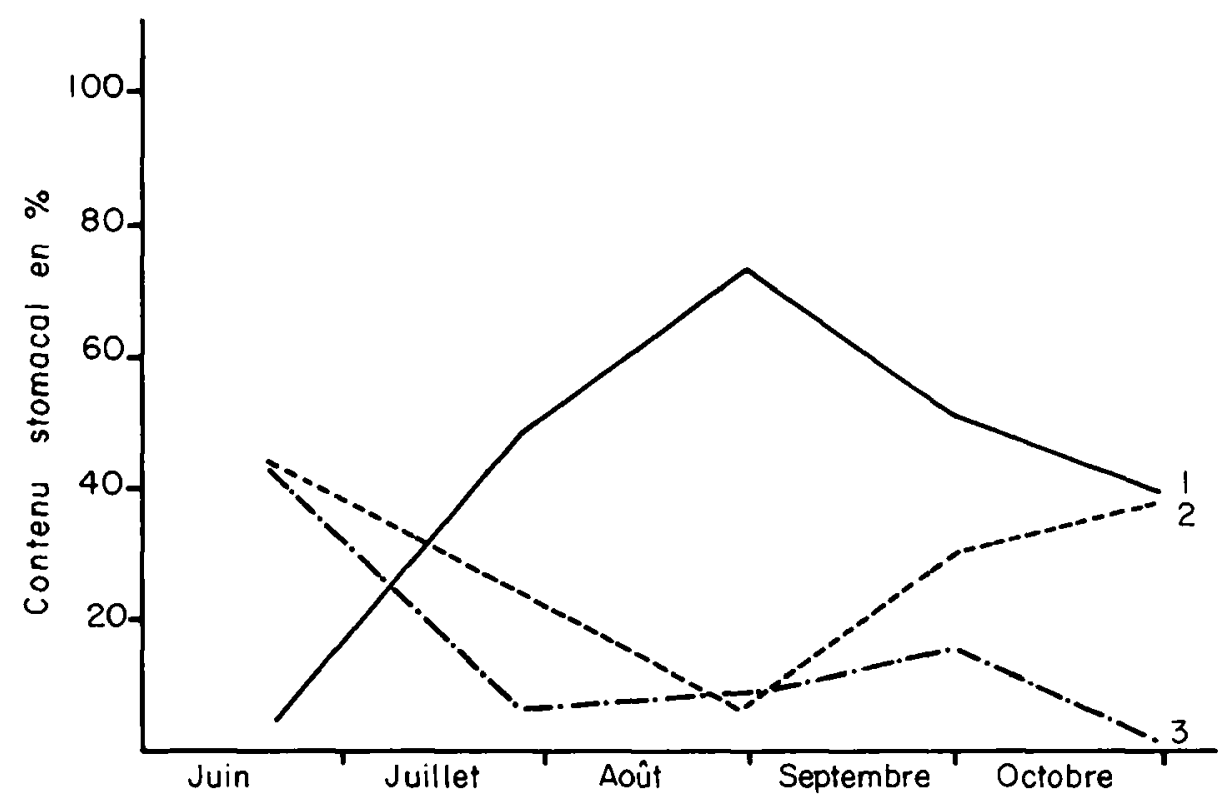

Figure 19 : Evolution du contenu stomacal chez Coregonus albula au cours de l'année. (ENDERLEIN, 1981).

1 : Bosmina coregoni maritima

2 : Eurytemora sp

3 : Limnocalanus grimaldii.

matin des Limnocalanus, qui est une espèce à sténotherme froid (au fond), à midi, il ne se nourrit pas et le soir il consomme surtout des bosmines et des eurytemora, qui sont également des espèces migrant du fond vers la surface le soir (ENDERLEIN, 1981). La migration verticale peut aussi dépendre de la température car BIKKININ (1978) signale que Coregonus lavaretus migre en zone plus profonde lorsque la température de l'épilimnion atteint $15-16^{\circ} \mathrm{C}$. D'autre part, cette sélection des espèces mangées est directe. ment liée à la vision du prédateur. Les bosmines, qui sont très pigmentées, constituent des proies très visibles: par contre, certaines daphnies transparentes ou les petites daphnies sont très rarement retrouvées dans l'estomac des poissons (ENDERLEIN. 1981 ).

L'intensité de prise de nourriture change au cours de l'année pour le Coregonus albula. Elle représente plus de $30 \%$ du poids sec du poisson par 24 heures en juillet et descend a 2-3\% en juin et octobre. Le taux d'évacuation gastrique est d'ailleurs très rapide : $50 \%$ de l'aliment consommé a quitté l'estomac après 1 h 20 minutes (ENDERLEIN, 1981). Les préférences alimentaires de quelques espèces sont résumées dans le tableau $n^{\circ} 10$.

\begin{tabular}{|l|c|c|c|c|c|}
\hline & C. clupeaformis & C. muksun & C. albula & C. peled & C. lavaretus \\
\hline $\begin{array}{l}\text { Alimentation : } \\
\text { - crustacés } \\
\text { insectes } \\
- \text { larves d'insectes } \\
- \text { mollusques } \\
- \text { poissons }\end{array}$ & $* *$ & $* * * *$ & $* * * *$ & $* * * *$ & $* *$ \\
\hline
\end{tabular}

Tableau 10 : Résumé des préférences alimentaires de quelques espèces de corégones.

\section{3) La temperature}

Les corégones exigent, normalement, de l'eau froide (inférieure à $25^{\circ} \mathrm{C}$ ), trans- 
parente (2 m au moins) et saturée en oxygène (20-50\%) (KOSIKOWSKA, 1976). La température létale pour le Coregonus hovi varie, suivant la température préalable d'acclimatation, de $22,4^{\circ} \mathrm{C}$ (acclimatation à $0^{\circ} \mathrm{C}$ ) à $26,7^{\circ} \mathrm{C}$ (acclimatation à $22,4^{\circ} \mathrm{C}$ ) (EDSALL et al., 1970).

\section{4) Reproduction}

\section{a) Age de la puberté}

Le Coregonus clupeaformis atteint la maturité sexuelle dans les lacs Hélène et Nathalie à un poids de $500 \mathrm{~g}$ ( 8 ans), pour le mâle, et de $600 \mathrm{~g}$ ( 9 ans) pour la femelle (DUMONT et FORTIN, 1978). Mais, dans d'autres endroits, comme à Chunozero (U.R.S.S.), il atteint la maturité sexuelle à un poids de $260 \mathrm{~g} \mathrm{(5} \mathrm{ans)} \mathrm{pour} \mathrm{le} \mathrm{mâle,} \mathrm{et} \mathrm{de}$ $290 \mathrm{~g}$ (6 ans) pour la femelle (RESHETNIKOV, 1966).

Le Coregonus peled, lui, est pubère à partir de 2 ans (poids de 400 à $500 \mathrm{~g}$ ) (VOLGIN, 1973 ; KANEP, 1973). Le mâle de lavaret est pubère à 2 -3 ans et la femelle à 3-4 ans (BAGENAL, 1970 ; STEFFENS, 1978). Le plus grand nombre de femelles de Coregonus peled se reproduisent lorsque la température de l'eau se situe entre 0,7 et $2,4^{\circ} \mathrm{C}$.

\section{b) La gamétogenèse}

La gamétogenèse commence lorsque la température est de 2,1 à $1,2^{\circ} \mathrm{C}$ chez les jeunes ( 2 à 3 ans) et de 1.2 a $0,9^{\circ} \mathrm{C}$ chez les sujets de plus de 4 ans. Les testicules représentent $0,03 \%$ du poids corporel en moyenne, à la fin de la première année (immature), 0,33 à $1,88 \%$, à la fin de la deuxième année et 0,52 à $2,50 \%$ à la fin de la troisième année ( $82 \%$ des mâles étant matures à 2 ans) (fig. 20). De même chez

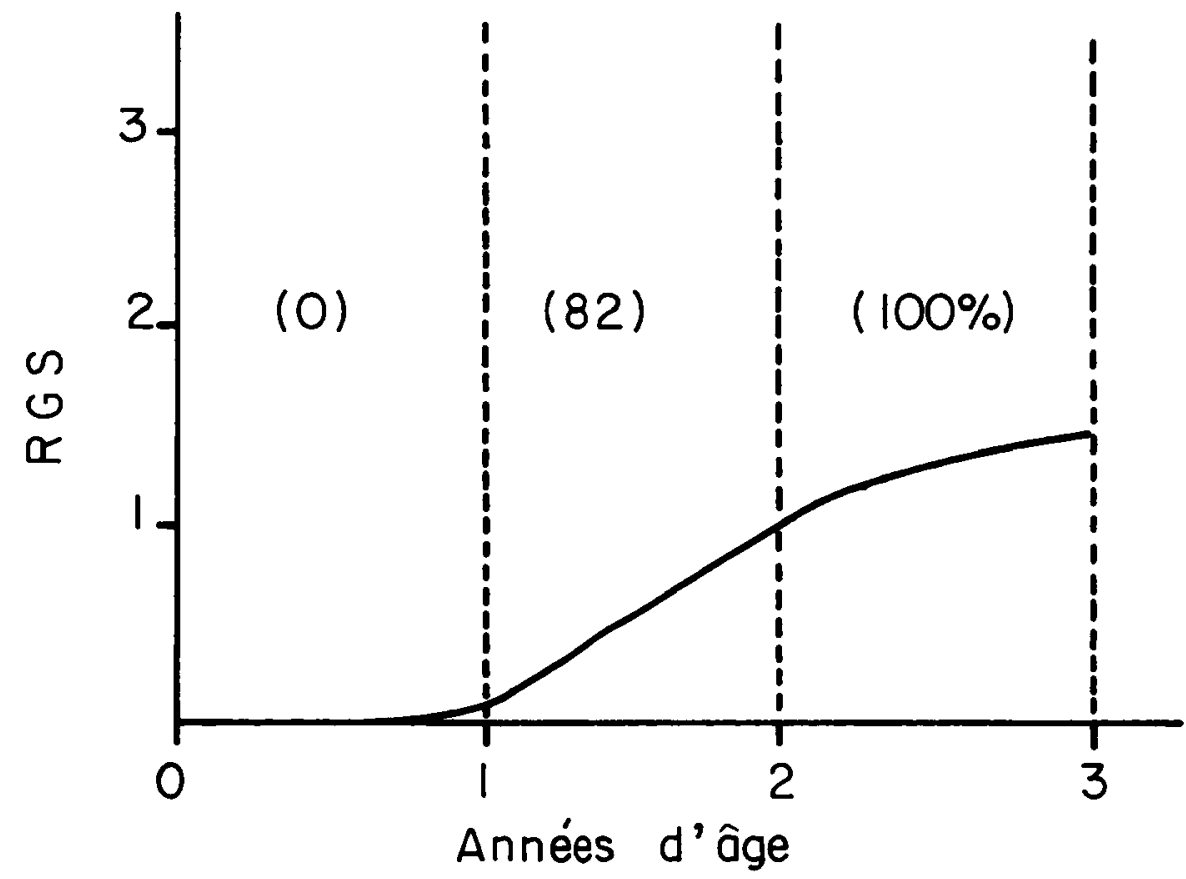

Figure 20 : Poids des testicules en pourcentage du poids du corps en fonction de I'âge chez le Coregonus lavaretus. (HOCHMAN et JIRASEK, 1975) ()\% de mâles pubères dans la population

Coregonus peled, cela nous donne respectivement 0,03 à $0,05 \%, 0,32$ à $1,64 \% ; 0,8$ a $1,77 \%$ et 1,02 à $1,86 \%$ au cours des 4 premières années $(94 \%$ des mâles étant matures à 2 ans) (HOCHMAN et JIRASEK, 1975). Le RGS apparaît donc particulièrement faible chez le mâle. 
Chez la femelle du Coregonus peled, le poids des gonades représente $0,15 \%$ du poids total à l'âge de 1 an, $13,05 \%$ à 2 ans, $14.61 \%$ à 3 ans, et $15.61 \%$ à 4 ans (poisson pubère à 2 ans) (HOCHMAN ET JIRASEK, 1977). Chez le Coregonus lavaretus maraena Bloch, on obtient les chiffres suivants : $11.76 \%$ a 2 ans, $15,77 \%$ a 3 ans et $20.32 \%$ à 4 ans (30\% des poissons étant pubères à 2 ans) (fig. 21) (HOCHMAN, 1966). Le RGS de la

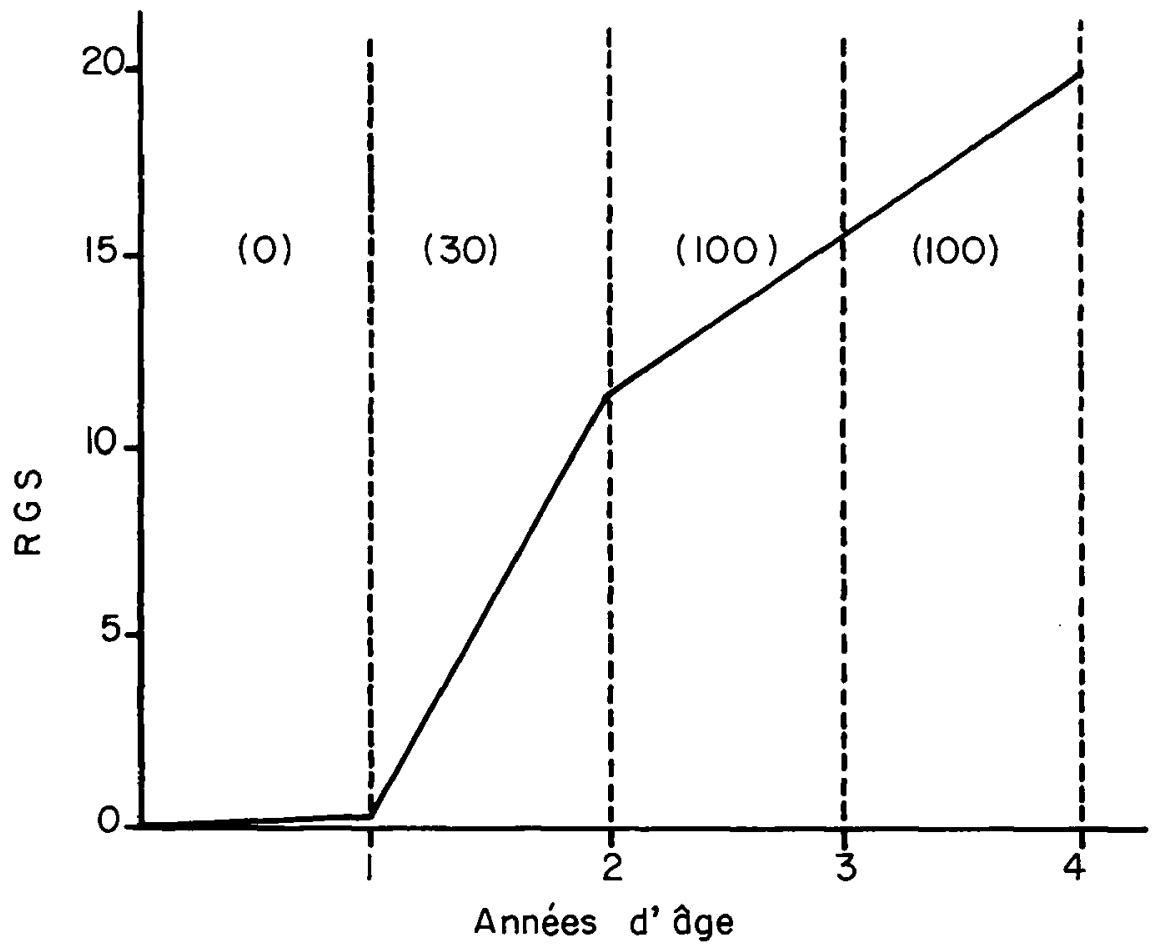

Figure 21 : Poids des ovaires en pourcentage du poids du corps en fonction de l's 80 chez le Coregonus lavaretus maraena Bloch. (HOCHMAN, 1966) () \% individus atteignant la maturité sexuelle.

femelle est beaucoup plus élevé que chez le mâle et s'apparente aux données rapportées pour d'autres espèces. La possibilité d'un " silence " reproducteur, certaines années chez l'adulte, a été discutée par MOREAU (1981) et KURLIN (1979).

\section{c) Production et qualité du sperme}

Coregonus lavaretus maraena Bloch, comme Coregonus peled, montre à 3-4 ans une courte mais intensive période de spermiation. Les sujets de 300 a $600 \mathrm{~g}$ ( 2 a 3 ans) ont une période de spermiation plus longue. Si on prélève le sperme de Coregonus lavaretus maraena Bloch à plusieurs reprises (5 à 6 fois), on obtient, au total, pour des mâles de $400 \mathrm{~g}(326 \mathrm{~mm})$, une production de laitance de $5,61 \mathrm{ml}(5,83 \mathrm{~g})$, soit $9,3 \mathrm{ml} / \mathrm{kg}$ de poids corporel (HOCHMAN et PENAZ, 1970).

Chez le Coregonus peled, on obtient, au total, pour des mâles de $600 \mathrm{~g}$, après 3 prélèvements (il faut respecter un minimum de 5 jours entre chaque prélèvement) : 2,5 $\mathrm{ml}$, soit $4,25 \mathrm{ml} / \mathrm{kg}$. On obtient donc 2 fois moins de sperme chez le Coregonus peled que chez le Coregonus lavaretus maraena Bloch (HOCHMAN et al., 1974). La production de sperme augmente progressivement au début de la période de spermiation (fig. 22). Chez ces deux espèces, la concentration en spermatozoïdes est faible, de l'ordre de 7,013 3 $10^{6}$ spermatozoïdes par $\mathrm{ml}\left(2,4\right.$ a $14,2 \times 10^{6}$ spermatozoïdes par $\mathrm{ml}$ selon l'époque). HOCHMAN et al.(1974) conseillent d'utiliser plusieurs mâles pour féconder une femelle.

La qualité du sperme a été estimée d'après la durée et l'intensité de motilité. On peut différencier quatre types de mouvement du sperme :

1. mouvement intensif de tout le sperme pendant environ $0,5 \mathrm{mn}$ : 


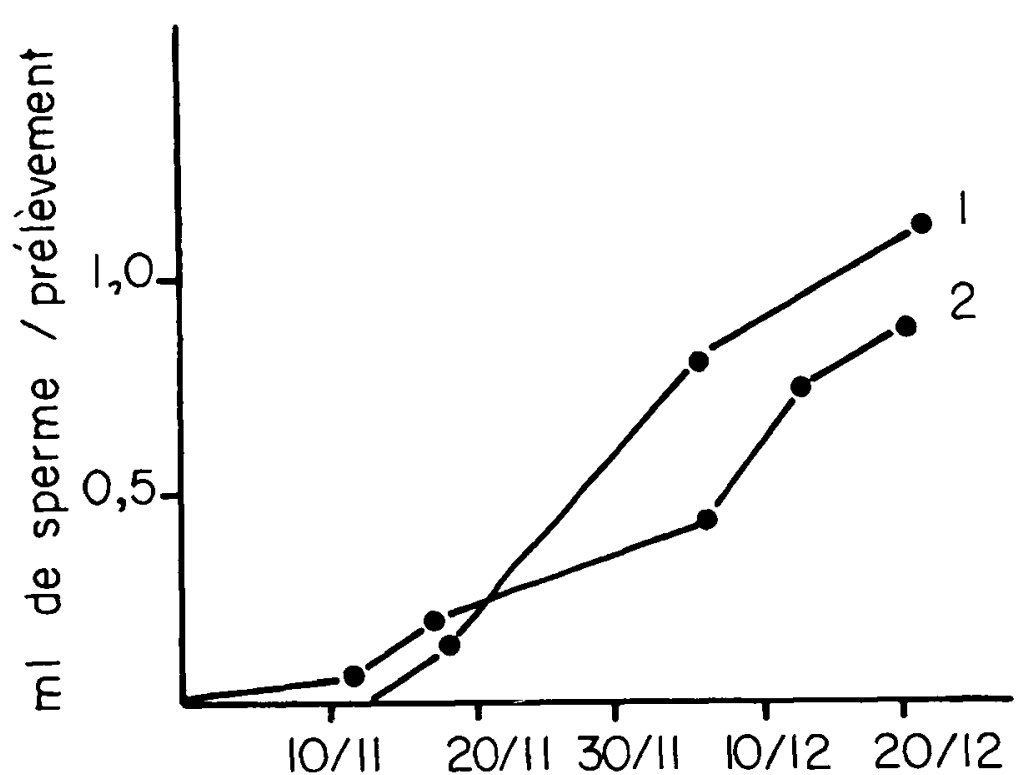

Figure 22 : Evolution de la production de laitance au début de la période de spermiation chez $C$. peled.

1 : mâles $1^{+}$, poids moyen $449 \mathrm{~g}(\mathrm{n}=23)$ étang de la région de Namest 2 : mâles $1^{+}$, poids moyen $693 \mathrm{~g}(\mathrm{n}=38)$ étang de la région de Lipnice, (d'après KOCHMAN et al., 1974).

2. mouvement intermédiaire pendant environ $1 \mathrm{mn}$;

3. mouvement modéré qui se prolonge pendant $1.5 \mathrm{mn}$ environ ;

4. mouvement sur place pendant à peu près $4 \mathrm{mn}$;

On a, pour une température de l'eau de 2,1 a $3,5^{\circ} \mathrm{C}$, une durée maximale du mouvement de $1.7 \mathrm{mn}$ chez Coregonus lavaretus maraena Bloch (HOCHMAN, 1966). Chez Coregonus peled, on obtient les chiffres suivants : $1: 0,31 \mathrm{mn}: 2: 0,49 \mathrm{mn} ; 3: 0,74 \mathrm{mn}$ : $4: 1.61 \mathrm{mn}$. On remarque donc une durée maximale du mouvement de $0.74 \mathrm{mn}$, ce qui est très court. De plus, it y a en moyenne $27,5 \%$ de spermatozoides immobiles dès la mise du sperme dans l'eau (HOCHMAN et al., 1974). Chez Coregonus autumnalis migratorius la quantité du sperme collecté et sa concentration dépendent de l'âge des mâles, mais la qualité (motilité) ne varie pas (tableau $n^{\circ} 11$ ). La production de sperme

\begin{tabular}{|c|c|c|c|c|c|c|}
\hline \multirow[t]{2}{*}{$\begin{array}{l}\text { Age des } \\
\text { mâles }\end{array}$} & \multirow[t]{2}{*}{$n$} & \multirow[t]{2}{*}{$\begin{array}{c}\mathrm{ml} \text { de sperme } \\
\text { par éjaculat }\end{array}$} & \multirow{2}{*}{$\begin{array}{c}{[\mathrm{C}] \text { en }} \\
\text { spermatozoïdes } \\
10^{9} \times \mathrm{ml}\end{array}$} & \multirow[t]{2}{*}{$\begin{array}{c}\text { Durée de } \\
\text { motilité en sec. }\end{array}$} & \multicolumn{2}{|c|}{$\begin{array}{l}\text { Volume de l'ejaculat } \\
\text { en relation avec la charge }\end{array}$} \\
\hline & & & & & $200 / \mathrm{m}^{3}$ & $700 / \mathrm{m}^{3}$ \\
\hline $\begin{array}{l}6^{+} \\
7 \\
8 \\
9 \\
10 \\
11 \\
12 \\
13\end{array}$ & $\begin{array}{r}3 \\
21 \\
58 \\
82 \\
41 \\
15 \\
5 \\
1\end{array}$ & $\begin{array}{r}0,4 \pm 0,09 \\
0,43 \pm 0,05 \\
0,45 \pm 0,03 \\
0,51 \pm 0,03 \\
0,57 \pm 0,04 \\
0,75 \pm 0,05 \\
0,70 \pm 0,02 \\
0,22\end{array}$ & $\begin{array}{l}4,13 \pm 0,57 \\
6,70 \pm 0,63 \\
6,90 \pm 0,34 \\
8,30 \pm 0,30 \\
8,50 \pm 0,44 \\
7,00 \pm 0,60 \\
6,9=1,20 \\
7,0\end{array}$ & $\begin{array}{l}36 \pm 0.70 \\
37=1.67 \\
39 \pm 1.01 \\
37 \pm 0.67 \\
37 \pm 0.84 \\
36 \pm 1.31 \\
36=1.72 \\
32\end{array}$ & $\begin{array}{l}2.52 \\
3,74 \\
3,22 \\
4,77 \\
7,70 \\
6,80 \\
6,80\end{array}$ & $\begin{array}{l}2,25 \\
2,36 \\
2,13 \\
2,60 \\
5,02 \\
5,02 \\
1,80\end{array}$ \\
\hline
\end{tabular}

Tableau 11 : Production et qualité du sperme de $C$. autumnalis migratorius prélevé sur des mâles d'âges différents (d'après KHODZER, 1981).

semble par contre dépendante de la densité de stockage des mâles (tableau n०11) KHODZHER (1981) constate aussi que le volume collecté et la concentration sont plus 
élevés au milieu de la période de spermiation: par contre. la durée de la motilité reste constante pendant toute la période et ne dépend pas non plus de la concentration.

\section{d) Fécondité relative}

Les valeurs rencontrées dans la littérature varient fortement selon les espèces mais aussi selon le stade considéré (ovocytes ou ovules), le taux d'exploitation (HEALEY. 1978), les conditions de milieu, l'âge des femelles, la latitude, le lac d'origine, etc., la relation n'étant pas linéaire entre poids et fécondité (HEALEY et NICOL, 1975).

En Italie (lac Majeur), pour Coregonus species, SPREAFICO et al. (1974) font état d'une fécondité de 44000 à 55000 cufs $/ \mathrm{kg}$. D'autres valeurs sont données dans le tableau $n^{\circ} 12$. Les données du Coregonus clupeaformis sont tirées des articles de

\begin{tabular}{|c|c|c|c|c|}
\hline Reproduction & C. clupeaformis & C. muksun & C. peled & C. lavaretus \\
\hline $\begin{array}{l}\text { - âge à la puberté } \\
\text { - poids des testicules } \\
\text { (\% du poids du corps) } \\
\text { - poids des ovaires } \\
\text { (\% du poids du corps) } \\
\text { - durée du mouvement des } \\
\text { spermatozoïdes (en s.) } \\
\text { - production de sperme (plusieurs } \\
\text { prélèvements en } \mathrm{ml} / \mathrm{kg} \text { ) } \\
\text { - concentration en spermato- } \\
\text { zoïdes }\left(10^{6} / \mathrm{ml}\right) \\
\text { - fécondité relative (nombre } \\
\text { d'ovules par } \mathrm{kg} \text { ) } \\
\text { - diamètre des ovules (mm) }\end{array}$ & $\begin{array}{c}14300 \text { à } \\
27600 \\
2 \text { à } 2,7\end{array}$ & $\begin{array}{c}5-6 \text { ans } \\
1,65 \\
15,4 \\
50 \\
\\
2,1-2,65 \text { (1) }\end{array}$ & $\begin{array}{c}2 \text { ans } \\
1,5 \\
14,61 \\
44 \\
14 \\
\\
40772 \mathrm{a} \\
87952\end{array}$ & $\begin{array}{c}2-3 \text { ans } \\
2 \\
15,77 \\
102 \\
4,25 \\
013 \quad 0 \text { à } \\
10 \text { 000 a } \\
21 \text { o00 } \\
3 \text { à } 3,7\end{array}$ \\
\hline
\end{tabular}

(1) KUKL.IN, 1979

Tableau 12 : Résumé de quelques caractéristiques concernant la reproduction de plusieurs espèces de corégones.

DUMONT et FORTIN (1978), OUADRI (1968), BRYAN et KATO (1975), BIDGOOD (1974). RESHETNIKOV (1966); celles du Coregonus peled de HOCHMAN et JIRASEK (1977), KANEP (1973); celles du Coregonus lavaretus de HUET (1972), FEDOROVA (1982): celles du Coregonus muksun de KUKLIN (1979).

\section{5) La fraie}

Les corégones fraient sur des fonds de sable, de gravier ou de roche. Le grand corégone (Coregonus clupeaformis) peut frayer d'octobre à janvier (BIDGOOD, 1974), mais la reproduction s'effectue généralement de fin octobre à fin décembre. Ils fraient généralement à une profondeur de 2-4 mètres (DUMONT et FORTIN, 1978), ou moins de 5 mètres, sur fond de gros cailloux ou blocs de pierres (MACHNIAK, 1975). Cependant, le Coregonus lavaretus semble, lui, frayer vers 7 mètres (BAGENAL, 1970).

La durée de la période de fraie d'une femelle ne dépasse pas généralement 10 jours (HUET, 1972). La fraie ne dépend pas exclusivement de la température (KOBLING, 1974), mais elle s'effectue dans de l'eau à une température inférieure à $8^{\circ} \mathrm{C}$, en eau libre ou sous couvert de glace (BIDGOOD, 1974). Le Coregonus peled, par exemple, fraie lorsque la température de l'eau est de 2 à $3^{\circ} \mathrm{C}$ (HOCHMAN et JIRASEK, 1977) et la meilleure production d'œufs a été obtenue chez le Coregonus lavaretus maraena Bloch lorsque la température de l'eau se situait entre 6 et $2^{\circ} \mathrm{C}$ (HOCHMAN, 1966).

II n'y a pas de changements anatomiques chez la femelle pendant cette période (Coregonus clupeaformis), mais il y en a quelques-uns chez le mâle qui devient rugueux avec des tubercules nuptiaux sur la tête. Pendant la période de fraie, le poisson ne s'alimente pas et nage au fond. II saute de l'eau au coucher du soleil et la nuit. La fraie a probablement lieu la nuit (QUADRI, 1968). 


\section{REMERCIEMENTS}

Nous remercions vivement le service de Documentation ( $M^{\prime l}$ Le HELEY), pour la recherche bibliographique effectuée. Monsieur DUMONT, de MONTREAL, a bien voulu relire le manuscrit dont la frappe a été assurée par Madame SANZ.

Ce travail a été réalisé dans le cadre d'une ATP INRA " Connaissance et gestion des écosystèmes lacustres et subalpins ".

\section{REFERENCES}

ALEKSANDROVA YE.N., 1973. The feeding of the local form of the Muksun (Coregonus muksun pallas) of the Lena River in connection with some of its morphological characteristics. J. Ichythyol., 13, 953-956

ANWAND K., 1976. Untersuchungsergebnisse über die Hechterbrütung Maränen. Setzlingsaufzucht und Glasaalhaälterung aus der Volksrepublick Polen. Z. Binnenfisch., 23, 344-237.

BAGENAL T.B., 1970. Note on the biology of the Schelly, Coregonus lavaretus (L), in Haweswater and Hullswater. J. Fish. Biol., 2, 137-154.

BATURO B., 1979. The health conditions of peled fry (Coregonus peled. Gmelin 1788) cultivated in the illuminated lake cages. Inland Fisheries Institute bl.5, 10, 957 olsztyn 5.

BEHNKE R.J., 1970. The application of cytogenetic and biochemical systematic to phylogenetic problems in the family salmonidae. Trans. Amer. Fish. Soc., 1, 237-248.

BENAROUS S., 1981. Perches (Perca fluviatilis) et corégones (Coregonus species) : étude du succès de la fraye et de l'embryogénèse dans le Léman français. Mémoire de I'E.N.I.T.A. de Dijon.

BIDGOOD B.F., 1974. Reproductive potential of 2 lake whitefish (Coregonus 'speaformis) populations. J. Fish. Res. Board Can., 31, 1631-1639.

BIKKININ R.F., 1978. Feeding and rations of whitefish Coregonus lavaretus in Lake Bannoye (Southern Urals). Hydrobiol. J., 14, 103.

BOGDANOVA, 1980. The development and feeding of the larvae of the lake Syamozera whitefish. Coregonus lavaretus pallosi $m$. exilis, under different temperature conditions and feeding regimes, J. Ichthyol., 20, 66-73.

BRAUM E., 1967. The survival of fish larvae with reference to their feeding behavior and the food supply. Pages 113-131 in S.D. GERKING, ed. The Biological basis of fish production, Blackwell Scientific Publications, Oxford.

BRAUM E., 1978. Ecological aspects of the survival of fish eggs, embryos and larvae. Pages 102-136, in S.D. GERKING, ed. Ecology of freshwater fish production. John Wiley and Sons, New York.

BROOKE L.T., 1975. Effect of different constant incubation temperatures on egg survival and embryonic development in lake whitefish (Coregonus clupeaformis). Trans. Amer. Fish. Soc., 104, 555-559.

BROOKE L.T., COLBY P.J., 1980. Development and survival embryos of lake herring at different constant oxygen concentrations and temperatures. Prog. Fish Cu/t., 42, 3-9.

BRYAN J.E., KATO D.A., 1975. Spawning of lake whitefish, Coregonus clupeaformis, and round whitefish, Prosopium cylindraceum, in Aishihik lake and east Aishihik River, Yukon territory, J. Fish. Res. Board Can., 32, 283-288.

BRYLINSKI E., RADZIEJ J., URYN B., 1979. Rearing of whitefish (Coregonus lavaretus L.) larvae and fry in illuminated lake cages. Roszniki Nauk. Rolniczych, seria H 99, 55-77.

CHERNYAEV Zh.A., 1982. La reproduction du Coregonus autumnalis migratorius. Moscou, Ed. Ind. Légère et Alimentaire.

CHRISTIE W.J., 1963. Effects of artificial propagation and the weather on recruitment in the Lake Ontario Whitefish Fishery. J. Fish. Res. Board Can., 20, 597-598. 
COLBY P.J., BROOKE L.T., 1973. Effects of temperature on embryonic development of lake herring (Coregonus artedii). J. Fish. Res. Board Can., 30, 799-810.

DUMONT P., FORTIN R., 1978. Quelques aspects de la biologie du grand corégone (Coregonus clupeaformis) des lacs Hélène et Nathalie, territoire de la baie James. Can. J. Zool., 56, 1402-1411.

EDSALL T.A., ROTTIER D.V., BROUND E.H., 1970. Temperature tolerance of bloater (Coregonus hoyi) J. Fish. Res. Board Can., 27, 2047-2052.

EDSALL T.A., ROTTIER D.V., 1976. Temperature of young of the year lake whitefish, Coregonus clupeaformis. J. Fish. Res. Board Can., 33, 177-180.

EINSELE W., 1965. Problems of fish larvae survival in nature and the rearing of economically important middle european freshwater fishes. Cal. Coop. Oceanid Fish. Inv. Rep., 10, 24-30.

ENDERLEIN O., 1981a. Interspecific food competition between the three pelagic zooplanctonfeeders, cisco (Coregonus albula L.). smelt (Osmerus eperlanus L), and herring (Clupea harengus L), in the Norrbotten part of the Bothnian Bay. Inst. Freshwater Research. Drottningholm, 59, 15-20.

ENDERLEIN O., 1981 b. When, where, what, and how much does the adult cisco, Coregonus albula $\mathrm{L}$, eat in the Bothnian Bay during the ice-free season. Inst. Freshwater Research. Drottningholm., 59, 21-32.

FEDOROVA G.V., 1982. Fecundity of the ladoga lake whitefish, in Les particularités biologiques des reproducteurs d'espèces de poissons à forte valeur économique, n० 178, 91-96, GOSNIORKH, Leningrad.

FLUCHTER J., 1980. Review of the present knowledge of rearing whitefish (Coregonidae) larvae. Aquaculture, 19, 191-208.

GRYGIEREK E., JANUSYKO M., WASILEWSKA E., 1980. Production de nourriture naturelle dans les étangs. Instytut Rybactwa Srodladowego, 132, 1-18.

GUNKEL G., KAUSCH H., 1979. Feeding and growth of fry and fingerlings whitefish, Coregonus fera J., E.I.F.A.C., Tech. Pap., 35, 163-176.

HALE J.G., 1970. Culture of lake herring in the laboratory. Progr. Fish Cult., 32, 217-221.

HEALEY M.C., 1978. Fecundity changes in exploited population of lake whitefish (Coregonus clupeaformis) and lake trout (Salvelinus namaycush)., J. Fish. Res. Board Can., 35, 945-950.

HEALEY M.C., NICOL C.W., 1975. Fecundity comparisons for various stocks of lake whitefish, Coregonus clupeaformis. J. Fish. Res. Board Can., 32, 404-407.

HOAGMAN W.J., 1973. Vital activity parameters as related to the early life history of larval and post-larval lake whitefish (Coregonus clupeaformis), 555-557, in J.H.S. BLAXTER Ed. The Early Life History of Fish, Springer Verlag Berlin Heidelberg New York.

HOCHMAN L., 1966. Reproductions properties of Coregonus lavaretus maraena (Bloch) in pond culture. Sb. V.S.Z., Brno, A., 453-468.

HOCHMAN L., PENAZ M., 1970. The volume of milt and vitality of sperms in Coregonus lavaretus maraena Bloch from pond culture. Zool. Listy.. 19, 281-292.

HOCHMAN L., PENAZ M., PROKES M., 1974. The volume of milt, quantity and quality of sperm in Coregonus peled (Gmelin, 1788) from pond culture. Zool. Listy., 23, 367-380.

HOCHMAN L., JIRASEK J., 1975. The size of male gonads in great maraena and in peled. Zivocisna Vyroba (Praha), 20, 887-896.

HOCHMAN L., JIRASEK J., 1977. The fertility of Coregonus peled Gmelin under pond conditions. Zivocisna Vyroba., 22, 839-848.

HOCHMAN L., SUKOP I., KLAS M., 1978. Food of the crossbed fry of the maraena (Coregonus lavaretus $\mathrm{L}$ ) and peled (Coregonus Peled $\mathrm{Gm}$ ). Zivocisna Vyroba., 23, 861-870.

HUET M., 1972. Cultivation of coregonids. Textbook of fish culture, 163-166.

JAGER T., NELLEN W., 1980. Die Erprobung einer polnischen methode zum Vorstrecken von Maränen in Schleswig-Holstein. Arbeiten des Deutschen Fishereiverbandes. 30, 14-31. 
JAGER T., NELLEN W., 1981. Illuminated net cages for rearing fish larvae. World conference on aquaculture, 1981, Venise.

JOHN K.R., HASLER A.D., 1956. Some factors affecting the hatching of eggs and the survival of young shallow water cisco $C$. artedii Le Sueur, in lake Mendota, Wisconsin. Limnol. Oceanogr., 1, 176-194.

KANEP S.V., 1973. General features of the growth, maturation and fecundity of pelyad (Coregonis peled Gm). J. Ichthyol., 13, 79-86.

KHODZHER L. Ch., 1981. Sperm production by the Baikal omul, Coregonus autumnalis migratorius. J. Ichthyol., 21, 131-137.

KOKOVA V. YE., 1978. The feeding of larvae of the whitefish, Coregonus lavaretus ludoga, the Baikal omul, Coregonus autumnalis migratorius, and the peled, Coregonus peled. K. Ichthyol., 18, 953-959.

KOBLING A., 1974. Der Starnberger See and die seinem Tröphiezustand angemessene Bewirtschaftungsweise des Coregonenbestandes. Veröff. Zool. Staatssamme. München, 17, 1-108.

KOZIANOWSKIA., 1979. Moderne Verfahren zur Coregonenaufzuch in der V.R. Polen Z. Binnenfisch. D.D.R., 26, 19-25.

KOSIKOWSKA S., 1976. Les corégones, leur biologie et la possibilité d'augmenter leur production. Rev. Trav. Inst. Pêches Marit, 40, 644-645.

KUKLIN A.A.. 1979. Maturation and reproduction of the Muksun, Coregonus muksun of the Yenisey River. J. Ichthyol., 19, 91-97

MACHNIAKK., 1975. The effects of hydroelectric development on the biology of northern fishes (reproduction and population dynamics) I. Lake Whitefish (Coregonus c/upeaformis Mitchill). A literature review and bibliography. Fish. Mar. Serv. Res. Dev. Tech. Rep., 527, 67pp.

MAECKLE H., 1975. On the artificial breeding of Coregonus wartmanni under raided pressure. Fischwirt, 25, 53-55.

MARCIAK Z., 1979. Food preference of juveniles of three coregonid species reared in cages. Cultivation of fish fry and its live food, European Mariculture Society Spec. Publ. 4, Bredene (Belgique), 127-137.

MARSHALL K.E., WOODS C.S., 1971. A bibliography of the coregonid fishes. Fisheries Research Board of Canada, Technical report $n^{\circ} 151$.

McCORNICK J.H., JONES B.R., SYRETTR.F., 1971. Temperature requirement for growth and survival of larval ciscos (Coregonus artedi). J. Fish. Res. Board Can., 28, 924-927.

MEDGYESY N., WIESER W., 1982. Rearing whitefish (Coregonus lavaretus) with frozen zooplankton by means of a new feeding apparatus. Aquaculture, 28, 327-337.

MOREAU G., 1981. Stratégie de reproduction chez les populations nordiques de Grands Corégones (Coregonus clupeaformis). Ver. Internat. Verein. Limnol., 21, 1251 1256.

QUADRI S.V., 1968. Growth and reproduction of the lake whitefish, Coregonus clupeaformis, in lake Ronge, Saskatchewan. J. Fish. Res. Board Can., 25, $2091-2100$.

RAISANEN G.R., BEHMER D.J., 1982. Rearing lake whitefish to fingerling size. Prog. Fish. Cult., 44, 33-35.

RECKAHN J.A., 1970. Ecology of young lake whitefish (Coregonus clupeaformis) in south bay, Manitoulin Island, Lake Huron, in C.C. HUNDREY and C.S. WOOD, Biology of coregonid fishes. Univ. of Manitoba Press.

RESHETNIKOV YU. S., 1966. Growth and maturation characteristics of whitefishes in northern water. Zakonomernosti dinamiki chislennosti ryb belogo morya i ego basseina, 93-155.

SCOTT W.B., CROSSMAN E.J., 1973. Freshwater fishes of Canada. Fisheries Research Board Canada, Ottawa, Bull. 184.

SPREAFICO E., BERG A., GRIMALDI E., 1974. Accrescimento e fecondita del coregone bondella (Coregonus $s p$ / considerati in rapporto alle modificazioni trofiche del Lago Maggiore. Mem. Ist. Ital. Idrobiol., 31, 205-220.

STEFFFNS W., 1978. Maränenzucht. Z. Binnenfisch, 25, 315-319, 327-331. 
SVARDSON G., 1945. Chromosome studies on Salmonidae., Inst. Freshw. Res. Drottingholm, Rept., 23, 1-151

SVARDSON G., 1949, 1957. The coregonid problem I-IV. Rept. Drottingholm Freshw. Fish. Res., 29, 89-101;31, 151-162;32, 79-125;33, 204-232;34, 141-164; 38, 267-356

SWANN C.G., DONALDSON E.M., 1980. Bibliography of salmonid reproduction 1963 1979 for the family Salmonidae: Subfamilies Salmonidae, Coregonidae and Thymallinae. Can. Techn. Rep. Fish. Aquat. Sci., n० 970.

TODD T.N., SMITH G.R., 1980. Differentiation in Coregonus zenithicus in Lake Superior. Can. Journ. Fish. Aquat. Sci., 37, no 12, 2228-2235.

URYN B.A., 1979. Farming of juvenile whitefish, Coregonus lavaretus (L.), in submerged illuminated cages. Cultivation of fish fry and its live food. European Mariculture Society. Spec. Publ., 4, 289-297.

VOLGIN M.V., 1973. Cultivation of marketable pelyad (Coregonus peled $\mathrm{Gm}$ ) in lake Titovo, Novosibisk province. J. Ichthyol., 13, 920-925

WOLF V., 1979. Versuche des Vorstreckens von Fischbrut in Gazekäfigen mit hilfe von Licht im V.E.B. Binnenfischerei Franckfurt (Oder). Z. Binnenfisch. D.D.R. 26 , 12-19.

YELL-TSOVA V.N., 1976. Predation on baikal omul (Coregonus autumnalis migratorius) larvae by common fishes in the Posl'skiy lagoon of Lake Baikal following their release from the Bol'shoya Rechka hatchery. J. Ichthyol., 16, 971-976.

YOCOM T.G., EDSALL T.A., 1974. Effect of acclimatation temperature and heat shock on vulnerability of fry of lake whitefish (Coregonus clupeaformis) to predation. $J$. Fish. Res. Board Can.. 31, 1503-1506. 